\title{
DERIVATION, $L^{\Psi}$-BOUNDED MARTINGALES AND COVERING CONDITIONS
}

\author{
BY
}

\author{
MICHEL TALAGRAND
}

\begin{abstract}
Let $(\Omega, \Sigma, P)$ be a complete probability space. Let $\left(\Sigma_{t}\right)_{t \in J}$ be a directed family of sub- $\sigma$-algebras of $\Sigma$. Let $(\Phi, \Psi)$ be a pair of conjugate Young functions. We investigate the covering conditions that are equivalent to the essential convergence of $L^{\Psi}$-bounded martingales. We do not assume that either $\Phi$ or $\Psi$ satisfy the $\Delta_{2}$ condition. We show that when $\Phi$ satisfies condition $\operatorname{Exp}$, that is when there exists an $a>0$ such that $\Phi(u) \leqslant \exp a u$ for each $u \geqslant 0$, the essential convergence of $L^{\Psi}$-bounded martingales is equivalent to the classical covering condition $V_{\Phi}$. This covers in particular the classical case $\Psi(t)=t(\log t)^{+}$. The growth condition Exp on $\Phi$ cannot be relaxed. When $J$ contains a countable cofinite set, we show that the essential convergence of $L^{\Psi}$-bounded martingales is equivalent to a covering condition $D_{\Phi}$ (that is weaker than $V_{\Phi}$ ). When $\Phi$ fails condition Exp, condition $D_{\Phi}$ is optimal. Roughly speaking, in the case of $L^{1}$-bounded martingales, condition $D_{\Phi}$ means that, locally, the Vitali condition with finite overlap holds. We also investigate the case where $J$ does not contain a countable cofinal set and $\Phi$ fails condition Exp. In this case, it seems impossible to characterize the essential convergence of $L^{\Psi}$-bounded martingales by a covering condition. Using the Continuum Hypothesis, we also produce an example where all equi-integrable $L^{1}$-bounded martingales, but not all $L^{1}$-bounded martingales, converge essentially. Similar results are also established in the derivation setting.
\end{abstract}

1. Results. Let $J$ be a directed set filtering to the right, i.e. a set of indices partially ordered by $\leqslant$, such that for each pair $t_{1}, t_{2}$ of elements of $J$ there exists an element $t_{3}$ such that $t_{1}, t_{2} \leqslant t_{3}$. A subset $J_{0}$ of $J$ is called cofinal if each element of $J$ is dominated by an element of $J_{0}$. Given a finite subset $I$ of $J$, and $t$ in $J$, we write $I \leqslant t$ if $t$ is greater than each element of $I$, and $t \leqslant I$ if each element of $I$ is greater than $t$.

Let $(\Omega, \Sigma, P)$ be a probability space. Sets and random variables are considered equal if they are equal almost surely. All sets and functions considered are measurable unless otherwise specified. Let $X=\left(X_{t}\right)_{t \in J}$ be a family of random variables taking values in $\overline{\mathbf{R}}$. The essential upper limit of $X$, e-limsup $X_{t}$, is defined by

$$
X^{*}=\text { e-limsup } X_{t}=\underset{s}{\operatorname{ess} i n f}\left(\underset{t \geqslant s}{\operatorname{ess} \sup } X_{t}\right)
$$

while the essential lower limit of $X$ is defined by

$$
X_{*}=\mathrm{e}-\liminf X_{t}=-\mathrm{e}-\limsup \left(-X_{t}\right) \text {. }
$$

Received by the editors October 2, 1984 and, in revised form, February 18, 1985.

1980 Mathematics Subject Classification. Primary 28A15, 60G40; Secondary 60G42.

Key words and phrases. Derivation, martingales, covering conditions, Orlicz spaces. 
The directed family $X$ is said to converge essentially if $X^{*}=X_{*}$ a.s. Since no other notion of convergence is used, we shall at times omit the word "essentially". For a family $A=\left(A_{t}\right)$ of sets indexed by $J$ we define $A^{*}$ by $1_{A^{*}}=$ e-limsup $I_{A_{t}}$.

A stochastic basis $\left(\Sigma_{t}\right)$ is an increasing family of sub- $\sigma$-algebras of $\Sigma$ indexed by $J$, i.e. for $s \leqslant t$ we have $\Sigma_{s} \subset \Sigma_{t}$. We denote by $E^{t}$ the conditional expectation with respect to $\Sigma_{t}$. A process $X$ is a family $\left(X_{t}\right)$ of random variables such that $X_{t}$ is $\Sigma_{t}$-measurable for every $t$. We say that $\left(X_{t}\right)$ is $L^{1}$-bounded if $\sup _{t} E\left|X_{t}\right|<\infty$; and that it is a martingale (resp. submartingale) if for $s \leqslant t, E^{s}\left(X_{t}\right)=X_{s}$ (resp. $\geqslant X_{s}$ ). A family $\left(A_{t}\right)$ of sets is adapted if $A_{t} \in \Sigma_{t}$ for every $t \in J$. A finite adapted family is an adapted family $A_{t}$ such that only finitely many of the sets $A_{t}$ are not empty.

An incomplete multivalued stopping time $\tau$ is a collection of sets $\{\tau=t\}$ for each $t \in J$, such that $\{\tau=t\} \in \Sigma_{t}$ and that the set

$$
R(\dot{\tau})=\{t \in J:\{\tau=t\} \neq \varnothing\}
$$

is finite and nonempty [8]. The set of incomplete multivalued stopping times is denoted by $I M$. For $\tau \in I M, t \in J$ we write $t \leqslant \tau$ if $t \leqslant R(\tau), \tau \leqslant t$ if $R(\tau) \leqslant t$. The sum function $S_{\tau}$ of $\tau \in I M$ is given by $S_{\tau}=\Sigma_{t} 1_{\{\tau \in t\}}$, and the excess function $e_{\tau}$ by $e_{\tau}=S_{\tau}-1_{\left\{S_{\tau} \geqslant 1\right\}}$. If $\tau \in I M$ and $A$ is an adapted family of sets, we set

$$
A(\tau)=\bigcup\left(\{\tau=t\} \cap A_{t}\right) .
$$

We now recall some classical facts about Orlicz spaces. References can be found in [12]. Let $\psi: \mathbf{R}^{+} \rightarrow \overline{\mathbf{R}}^{+}$be left continuous, nondecreasing zero at the origin. We set $\Psi(t)=\int_{0}^{t} \psi(u) d u$. Let $\phi$ be given by $\phi(u)=\sup \{s: \psi(s)<u\}$ for $u>0$. Let $\Phi(u)=\int_{0}^{u} \phi(t) d t$. Then $(\Phi, \Psi)$ are called conjugate Young's functions. For a measurable function $f$, let

$$
\|f\|_{\Psi}=\sup \{E(|f g|): E(\Phi(|g|)) \leqslant 1\} .
$$

We denote by $L^{\Psi}$ the space of those $f$ for which $\|f\|_{\Psi}<+\infty$. Provided with the norm $\|\cdot\|_{\Psi}$, it is a Banach lattice. We define $L^{\Phi}$ in a similar way. We have $L^{\Psi \cdot}=L^{\infty}$ if and only if $\Psi$ is always finite.

An essential fact is that conditional expectation with respect to a sub- $\sigma$-algebra is a norm-one operator on $L^{\Psi}$.

We shall use the following facts:

(1) If $\|f\|_{\Phi} \leqslant 1,\|g\|_{\Psi} \leqslant 1$, then $|E(f g)| \leqslant 1$.

(2) If $0<\|f\|_{\Phi}<\infty$, then $\int \Phi\left(|f| /\|f\|_{\Phi}\right) d P \leqslant 1$.

(3) $\|f\|_{\Phi} \leqslant 1+\int \Phi(|f|) d P$.

For the simplicity of the discussion, we shall assume that

(4) $\lim _{t \rightarrow \infty} \phi(t)=\infty$.

The only case that this assumption eliminates is the case $L^{\Psi}=L^{\infty}$, but this case is covered by the classical work of Krickeberg [5].

We say that a process $\left(X_{t}\right)$ is $L^{\Psi}$-bounded if $\sup \left\|X_{t}\right\|_{\Psi}<\infty$. Since we are interested in $L^{\Psi}$-bounded martingales, we can replace $\|\cdot\|_{\Psi}$ by an equivalent norm. Hence it is no loss of generality to assume that the following holds:

(5) $\Psi(t)>0$ and $\Phi(t)>0$ for $t>0$.

Without this unrestrictive hypothesis, the formulation of condition $D_{\Phi}$ (see Definition 3) would have to be more complicated. 
It is said that $\Psi$ satisfies the $\Delta_{2}$ condition if the following holds:

$$
\exists M>0, \forall u>1, \quad \Psi(2 u) \leqslant M \Psi(u) .
$$

Condition $\Delta_{2}$ holds if and only if $L^{\infty}$ is dense in $L^{\Psi}$ for the norm $\|\cdot\|_{\Psi}[6]$.

We shall need a growth condition that is weaker than condition $\Delta_{2}$. We say that $\Phi$ satisfies condition Exp if the following holds:

(Exp)

$$
\exists a>0, \forall u \geqslant 0, \quad \Phi(u) \leqslant \exp a u .
$$

Before we describe the covering-type conditions that we shall use, a comment is in order. The first thought of the reader glancing at these conditions is likely to be that no natural example of filtration (e.g. in multiparameter processes) seems to satisfy them. It is actually not a deep fact that a covering condition implies the convergence of certain types of martingales. Since multiparameter martingale convergences are deep facts, there is little hope to find easy proofs that the corresponding filtrations satisfy covering conditions. However, since this paper will show that covering conditions are actually necessary for martingale convergence, all the natural examples of filtrations actually satisfy some of the conditions we now state.

DEFINITION 1. We say that the stochastic basis $\left(\Sigma_{t}\right)$ satisfies condition $V_{\Phi}$ if for each adapted family $\left(A_{t}\right)$ and each $\varepsilon>0$ there exists $\tau \in I M$ with $P\left(A^{*} \backslash A(\tau)\right) \leqslant \varepsilon$ and $\left\|e_{\tau}\right\|_{\Phi} \leqslant \varepsilon$.

It is also possible to formulate condition $V_{\Phi}$ without using multivalued stopping times. In that language, $V_{\Phi}$ states that, for each adapted family $\left(A_{t}\right)$ and each $\varepsilon>0$, there exists an adapted family $\left(B_{t}\right)$, with $B_{t} \subset A_{t}$, all of the sets $B_{t}$ empty except finitely many ones, and such that $P\left(A^{*} \backslash \cup B_{t}\right) \leqslant \varepsilon$ and $\left\|\sum 1_{B_{t}}-1_{\cup B_{t}}\right\|_{\Phi} \leqslant \varepsilon$. The other covering conditions can be reformulated in a similar way.

Definition 2. We say that the stochastic basis $\left(\Sigma_{t}\right)$ satisfies condition $F V_{\Phi}$ if for each adapted family $A=\left(A_{t}\right)$ and each $\varepsilon>0$ there exist functions $\left(\xi_{t}\right)_{t \in J}, \xi_{t} \geqslant 0$, $\xi_{t}=0$ outside $A_{t}, \xi_{t}$ bounded, $\xi_{t}$ is $\Sigma_{t}$-measurable, such that only finitely many functions $\xi_{t}$ are not zero, and such that $\int\left(\sum \xi_{t}\right) d P \geqslant P\left(A^{*}\right)-\varepsilon$, and $\left\|\sum \xi_{t}-1 \wedge \xi_{t}\right\|_{\Phi}$ $\leqslant \varepsilon$.

Definition 3. We say that the stochastic basis $\left(\Sigma_{t}\right)$ satisfies condition $D_{\Phi}$ if one can write $\Omega$ as an increasing union $\Omega=\cup_{n} \Omega_{n}$, such that, for each adapted family $\left(A_{t}\right)$, each $n$ and each $\gamma>0$, there is $\tau \in I M$ with

$$
P\left(\left(A^{*} \cap \Omega_{n}\right) \backslash A(\tau)\right) \leqslant \gamma
$$

and $\int \Phi\left(e_{\tau} / n\right) d P \leqslant \gamma$.

DEFINITION 4. We say that the stochastic basis $\left(\Sigma_{t}\right)$ satisfies condition $D_{\Phi}^{\prime}$ if for each $\varepsilon>0$ there is $\eta(\varepsilon)>0$ such that, for each $\gamma>0$ and each adapted family of sets $\left(A_{t}\right)$, there is $\tau \in I M$ with $P\left(A^{*} \backslash A(\tau)\right) \leqslant \varepsilon$ and $\int \Phi\left(\eta(\varepsilon) e_{\tau}\right) d P \leqslant \gamma$.

Definition 5 . We say that the stochastic basis $\left(\Sigma_{t}\right)$ satisfies condition $C$ if for each $\varepsilon>0$ there is $M_{\varepsilon}>0$ such that, for each adapted family $A=\left(A_{t}\right)$ with $P\left(A^{*}\right)>\varepsilon$, there is $\tau \in I M$ with $P\left(A^{*} \backslash A(\tau)\right) \leqslant \varepsilon$ and $\left\|S_{\tau}\right\|_{\Phi} \leqslant M_{\varepsilon}$.

Condition $V_{\Phi}$ is classical. C. A. Hayes proved (in the setting of derivation [4]) that when $\Phi$ satisfies the $\Delta_{2}$ condition, condition $V_{\Phi}$ is necessary and sufficient for the convergence of $L^{\Psi}$-bounded martingales. Condition $F V_{\Phi}$ is a "function" version of condition $V_{\Phi}$, where the functions $1_{\{\tau=t\}}$ are replaced by more general functions. It is weaker than $V_{\Phi}$. Other trivial implications are $V_{\Phi} \Rightarrow D_{\Phi}^{\prime} \Rightarrow D_{\Phi} \Rightarrow C_{\Phi}$. 
In [10] A. Millet and L. Sucheston introduced a condition $C$, and they proved that this condition is sufficient for the convergence of $L^{1}$-bounded martingales. Our condition $C_{\Phi}$ is an obvious adaptation of their idea. They asked whether condition $C$ is necessary for the convergence of $L^{1}$-bounded martingales. This question, (communicated by L. Sucheston) to which we give a positive answer is at the origin of this work.

Our first result is of a very general nature.

THEOREM 6. Condition $F V_{\Phi}$ is sufficient for the convergence of $L^{\Psi}$-bounded martingales. If $L^{\Psi} \neq L^{1}$, condition $F V_{\Phi}$ is also necessary.

This result is not satisfactory, since condition $F V_{\Phi}$ involves functions instead of sets, so is not a Vitali-type condition. However, we shall see later that it is not readily possible to improve upon Theorem 6 without further hypothesis. But the situation is much better when $\Phi$ satisfies condition Exp.

THEOREM 7. When $\Phi$ satisfies condition Exp, condition $V_{\Phi}$ is necessary and sufficient for the convergence of $L^{\Psi}$-bounded martingales.

We can also improve Theorem 6 when the structure of $J$ is simple.

THEOREM 8. Condition $D_{\Phi}$ (resp. $\left.D_{\Phi}^{\prime}\right)$ is sufficient for the convergence of $L^{\Psi}$-bounded martingales. When $J$ contains a countable cofinal set, it is also necessary.

This theorem is proved in paragraph 4. Since the proof is long, we give a simpler argument for the important case $L^{\Psi}=L^{1}$ in paragraph 5. When $\Phi$ fails condition $\operatorname{Exp}, D_{\Phi}$ is the sharpest necessary condition that we know. This condition seems to be actually very sharp. In the case $L^{\Psi}=L^{1}$, an example of A. Millet and $L$. Sucheston shows that in general one cannot take the sets $\Omega_{n}$ in $\cup \Sigma_{t}[10]$. (Such an example could actually be produced whenever $\Phi$ fails condition Exp, along the lines of Theorem 10).

It can be useful to have whenever possible a sufficient condition that is simpler than condition $D_{\Phi}^{\prime}$.

THEOREM 9. Assume that $\Psi$ satisfies the $\Delta_{2}$ condition. Then condition $C_{\Phi}$ is sufficient for the convergence of $L^{\Psi}$-bounded martingales. (When $J$ contains a countable cofinal set, it is also necessary.)

The proof will show that actually the "function version" of $C_{\Phi}$ is also sufficient.

We now give results that show that the preceding cannot be readily improved upon. First, condition Exp in Theorem 6 cannot be relaxed.

ThEOREM 10. Assume that $\Phi$ fails condition Exp. Then there exist a countable index set $J$ and finite $\sigma$-algebras $\left(\Sigma_{t}\right)_{t \in J}$ of $[0,1]$ such that condition $D_{\Phi}$ holds (and hence $L^{\Psi}$-bounded martingales converge) but that condition $V_{\Phi}$ fails.

The filtration used in the proof of Theorem 10 is somehow artificial. It would be interesting to know what happens for "natural" filtrations. The same comment applies to Theorems 11 and 12 . 
It is not possible either to relax condition $\Delta_{2}$ in Theorem 9 .

TheOREM 11. Assume that $\Psi$ fails the $\Delta_{2}$ condition. Then there exists a countable index set $J$ and finite $\sigma$-algebras $\left(\Sigma_{t}\right)_{t \in J}$ on $[0,1]$ such that condition $C_{\Phi}$ holds but not all $L^{\Psi}$-bounded martingales converge essentially (and hence condition $D_{\Phi}$ fails).

One of the consequences of Theorem 7 is that when $\Phi$ satisfies condition Exp, the convergence of $L^{\Psi}$-bounded martingales is equivalent to $V_{\Phi}$, a covering type condition. The same conclusion holds from Theorem 8 , but this time under the hypothesis that $J$ contains a countable cofinal set. Does this conclusion always hold? The weakest useful covering type condition we know of is condition $C_{\Phi}$, so the following seems to show that the answer is no.

TheOREM 12. Assume that $\Phi$ does not satisfy condition Exp. Then there exist an index set $J$ and a stochastic basis of finite algebras $\left(\Sigma_{t}\right)_{t \in J}$ on $[0,1]$ that satisfy condition $F V_{\Phi}$ but fail condition $C_{\Phi}$.

It has been shown by $\mathrm{K}$. Astbury [1] that when $J$ contains a countable cofinal subset, the convergence of $L^{1}$-bounded martingales is equivalent to the convergence of equi-integrable $L^{1}$-bounded martingales. Using the Continuum Hypothesis, we show that this is not the case in general.

THEOREM 13. Under the Continuum Hypothesis, there exists a stochastic basis of finite algebras on $[0,1]$ such that all equi-integrable $L^{1}$-bounded martingales converge, but some $L^{1}$-bounded martingales fail to converge.

It is also possible, using the Continuum Hypothesis, to show that condition $F V_{\infty}$ (that is condition $F V_{\Phi}$ for $L^{\Psi}=L^{1}$ ) is not necessary for the convergence of $L^{1}$-bounded martingales, but we shall not do it here.

We now turn to derivation. It is essentially a routine to transform a proof of a theorem concerning martingales on an index set to a proof of the corresponding theorem concerning derivation, except that the exhaustion arguments are less straightforward (but nevertheless a standard technique). In order to keep this paper at a reasonable length, we have chosen to give only the proofs in the martingale case, except for Theorem 21 , that is proved in $\$ 10$.

Let $(\Omega, \Sigma, \mu)$ be a probability space. (We do not consider the case of infinite measure. Our results can be adapted to this case, but the statements are not so simple since we no longer have $L^{\infty} \subset L^{\Psi}$.) Suppose that for each $x$ in $\Omega$, we are given a family $\mathscr{B}(x)$ of Moore-Smith sequences (i.e. families filtering to the right) of measurable sets of positive measure, such that if a sequence belongs to $\mathscr{B}(x)$, the same holds for each of its cofinal subsequences. The collection $\mathscr{B}$ of the families $\mathscr{B}(x)$ is called a derivation basis. For $f \in L^{1}(\mu)$ we set

$$
D^{*} f(x)=\sup \left[\limsup \frac{\int_{A_{t}} f d \mu}{\mu\left(A_{t}\right)}\right]
$$


where the limsup refers to a family $\left(A_{t}\right)_{t \in J}$ in $\mathscr{B}(x)$ and the sup to the choice of $\left(A_{t}\right)$ in $\mathscr{B}(x)$. We define $D_{*} f(x)$ in a similar way, using inf instead of sup. We say that $\mathscr{B}$ differentiates $f$ if $D^{*} f(x)=D_{*} f(x)=f(x) \mu$ a.e. If $H$ is a subspace of $L^{1}$, we say that $\mathscr{B}$ differentiates $H$ if it differentiates each $f$ in $H$.

If for each point $x$ of a (not necessarily measurable) subset $X$ of $\Omega$ we are given a sequence $\left(A_{t}(x)\right)$ of $\mathscr{B}(x)$, we say that the collection $\mathscr{V}$ of these sequences is a Vitali cover of $X$, and we call a $\mathscr{V}$-set any set of the type $A_{t}(x)$.

Given a finite family $\mathscr{F}$ of measurable sets, we denote

$$
e_{\mathscr{F}}=\sum_{\mathscr{F}} 1_{A}-1 \wedge \sum_{\mathscr{F}} 1_{A} ; \quad d_{\mathscr{F}}=\bigcup_{\mathscr{F}} A
$$

DEFINITION 14. We say that $\mathscr{B}$ satisfies condition $V_{\Phi}$ if, for each $X \subset \Omega$, each Vitali cover $\mathscr{V}$ of $X$, and each $\varepsilon>0$, there exists a finite family $\mathscr{F}$ of $\mathscr{V}$-sets with $\left\|e_{\mathscr{F}}\right\|_{\Phi} \leqslant \varepsilon$ and $\mu^{*}\left(X \backslash d_{\mathscr{F}}\right) \leqslant \varepsilon$.

DEFINITION 15. We say that $\mathscr{B}$ satisfies condition $F V_{\Phi}$ if, for each $X \subset \Omega$, each Vitali cover $\mathscr{V}$ of $X$, and each $\varepsilon>0$, there exists a finite family $\mathscr{F}$ of $\mathscr{V}$-sets and for $A \in \mathscr{F}$ numbers $\alpha_{A} \geqslant 0$, such that if $\xi=\sum_{\mathscr{F}} \alpha_{A} 1_{A}$, we have $\|\xi\|_{1} \geqslant \mu^{*}(X)-\varepsilon$ and $\|\xi-\xi \wedge 1\|_{\Phi} \leqslant \varepsilon$.

Definition 16. We say that $\mathscr{B}$ satisfies condition $D_{\Phi}^{\prime}$ (resp. $C_{\Phi}$ ) if for each $\varepsilon>0$ there is $\eta>0$ (resp. $M_{\varepsilon}<\infty$ ) such that, for each $X \subset \Omega$, each Vitali cover $V$ of $X$, and each $\gamma>0$, there is a finite family $\mathscr{F}$ of $\mathscr{V}$-sets with $\mu^{*}\left(X \backslash d_{\mathscr{F}}\right) \leqslant \varepsilon$ and $\int \Phi\left(\eta e_{\mathscr{F}}\right) d P \leqslant \gamma\left(\right.$ resp. $\left.\left\|e_{\mathscr{F}}\right\|_{\Phi} \leqslant M_{\varepsilon}\right)$.

DEFINITION 17. We say that $\mathscr{B}$ satisfies condition $D_{\Phi}$ if one can write $\Omega$ as an increasing union of measurable sets $\Omega_{n}$, such that for each $X \subset \Omega$, each Vitali cover $V$ of $X$, and each $\gamma>0$, there is a finite family $\mathscr{F}$ of $\mathscr{V}$-sets with $\left.\mu^{*}\left(\left(\Omega_{n} \cap X\right)\right) \backslash d_{\mathscr{F}}\right)$ $\leqslant \gamma$ and $\int \Phi\left(e_{\mathscr{F}} / n\right) d P \leqslant \gamma$.

We then have

THEOREM 18. Condition $F V_{\Phi}$ is sufficient for $\mathscr{B}$ to differentiate $L^{\Psi}$. If $L^{\Psi} \neq L^{1}$, it is also necessary.

THEOREM 19. If $\Phi$ satisfies condition Exp, condition $V_{\Phi}$ is necessary and sufficient for $\mathscr{B}$ to differentiate $L^{\Psi}$.

THEOREM 20. Condition $D_{\Phi}^{\prime}$ is sufficient for $\mathscr{B}$ to differentiate $L^{\Psi}$. When $\Psi$ satisfies the $\Delta_{2}$ condition, condition $C_{\Phi}$ is also sufficient.

One could think at first that the usual hypothesis that the Moore-Smith sequences of sets in each $\mathscr{B}(x)$ are actual sequences would correspond to the hypothesis that the index set has countable cofinality in the martingale setting. This is not the case, as the following shows.

THEOREM 21. Suppose that $\Phi$ fails condition Exp. There exists a compact metric space $L$ and a derivation basis $\mathscr{B}$ on $L$, such that each $\mathscr{B}(x)$ consists of the subsequences of a sequence $W_{n}(x)$ of open sets of diameter going to zero, and such that $\mathscr{B}$ differentiates $L^{\Psi}$ but that condition $C_{\Phi}$ fails. 
Let $\mathscr{U}$ be a family of measurable sets of $\Omega$ and $\delta$ a function from $\mathscr{U}$ to $\mathbf{R}^{+}$. If for each $x \in \Omega, \mathscr{B}(x)$ consists of the sequences $\left(U_{n}\right)$ such that $x \in U_{n} \in \mathscr{U}$ and $\delta\left(U_{n}\right) \rightarrow 0$, we say that $\mathscr{B}$ is a $D$-basis [3]. It turns out that $D$-bases behave like martingales whose index set have a countable cofinality.

THEOREM 22. Suppose that $\mathscr{B}$ is a D-basis. Then condition $D_{\Phi}$ is necessary and sufficient for $\mathscr{B}$ to differentiate $L^{\Psi}$.

ACKNOWLEDGMENT. The author was introduced to these problems by Professor Sucheston, with whom he had several stimulating conversations.

2. Proof of Theorem 6. The proof that condition $F V_{\Phi}$ is sufficient for the convergence of $L^{\Psi}$-bounded martingales is routine, as will be the proof of the sufficiency part of Theorems 8 and 9 . We reproduce it for completeness.

Proposition 23. Assume that condition $F V_{\Phi}$ holds. Let $\left(X_{t}\right)$ be a positive submartingale that is $L^{\Psi}$-bounded. Let $s \in J$ and $B \in \Sigma_{s}$. Then for each $\lambda>0$ we have

$$
P\left(B \cap\left\{X^{*} \geqslant \lambda\right\}\right) \leqslant \frac{1}{\lambda} \sup _{t} E\left(X_{t} 1_{B}\right) .
$$

Proof. Let $k$ be such that $\left\|X_{t}\right\|_{\Psi} \leqslant k$ for each $t$. Let $0<\beta<\lambda$. For $t \geqslant s$, let $A_{t}=\left\{X_{t}>\beta\right\} \cap B$ and let $A_{t}=\varnothing$ otherwise. The family $\left(A_{t}\right)$ is adapted. We have $\left\{X^{*} \geqslant \lambda\right\} \cap B \subset A^{*}$.

Let $\varepsilon>0$. According to condition $F V_{\Phi}$, there is a finite set $I \subset J$, and for $i \in J$, there is a function $\xi_{i}>0, \xi_{i}$ bounded, $\xi_{i}$ zero outside $A_{i}$, where $\xi_{i}$ is $\Sigma_{i}$-measurable, such that $\int \Sigma_{I} \xi_{i} d P \geqslant P\left(A^{*}\right)-\varepsilon$ and $\left\|\xi^{\prime}\right\|_{\Phi} \leqslant \varepsilon$, where $\xi^{\prime \prime}=1 \wedge \Sigma_{I} \xi_{i}, \xi^{\prime}=\Sigma_{I} \xi_{i}-$ $\xi^{\prime \prime}$.

Let $t \in I$ with $I \leqslant t$. We can write

$$
\begin{aligned}
P\left(A^{*}\right)-\varepsilon & \leqslant E\left(\sum_{I} \xi_{i}\right)=E\left(\sum_{I} \xi_{i} 1_{A_{i}}\right) \leqslant \frac{1}{\beta} E\left(\sum_{I} \xi_{i} X_{i}\right) \leqslant \frac{1}{\beta} \sum_{I} E\left(\xi_{i} X_{i}\right) \\
& \leqslant \frac{1}{\beta} \sum_{I} E\left(\xi_{i} X_{t}\right)=\frac{1}{\beta} E\left(\sum_{I} \xi_{i} X_{t}\right)=\frac{1}{\beta} E\left(\xi^{\prime} X_{t}\right)+\frac{1}{\beta} E\left(\xi^{\prime \prime} X_{t}\right) .
\end{aligned}
$$

Since $\left\|\xi^{\prime}\right\|_{\infty} \leqslant 1$, and $\xi^{\prime}=0$ outside $B$, we get $E\left(\xi^{\prime} X_{t}\right) \leqslant E\left(X_{t} 1_{B}\right)$. Since $\left\|\xi^{\prime}\right\|_{\Phi} \leqslant \varepsilon$ and $\left\|X_{t}\right\|_{\Psi} \leqslant k$, we get $E\left(\xi^{\prime} X_{t}\right) \leqslant \varepsilon k$, so

$$
P\left(\left\{X^{*} \geqslant \lambda\right\} \cap B\right) \leqslant(1 / \beta) \sup E\left(X_{t} 1_{B}\right)+\varepsilon k / \beta .
$$

Letting $\varepsilon \rightarrow 0$, and then $\beta \rightarrow \lambda$, we get the result.

Proposition 24. Assume that for every $L^{\Psi}$-bounded positive submartingale $X_{t}$, every $s \in J$, every $B \in \Sigma_{s}$ and every $\lambda>0$, we have

$$
P\left(B \cap\left\{X^{*} \geqslant \lambda\right\}\right) \leqslant(1 / \lambda) \sup E\left(X_{t} 1_{B}\right) .
$$

Then every $L^{\Psi}$-bounded martingale converges.

Proof. The case of $L^{\Psi}=L^{1}$ will be covered by the more general Proposition 39, so we assume $L^{\Psi} \neq L^{1}$. In this case, an $L^{\Psi}$-bounded martingale is equi-integrable, so is of the type $X_{t}=E^{t}(f)$ for some $f \in L^{\Psi}$. Let $\varepsilon>0$. There is $h \in L^{\infty}$ with 
$\|f-h\|_{1}<\varepsilon^{2}$, so there is $s \in J$, and a bounded $g$ that is $\Sigma_{s}$-measurable, such that $\|f-g\| \leqslant \varepsilon^{2}$. Let $Y_{t}=E^{t}(|f-g|)$. This is a positive martingale, bounded in $L^{\Psi}$, and such that $E\left(Y_{t}\right) \leqslant \varepsilon^{2}$ for each $t$, so we have $P\left(Y^{*} \geqslant \varepsilon\right) \leqslant \varepsilon$. Since $\left|X_{t}-E^{t}(g)\right|$ $\leqslant Y_{t}$, and since $E^{t}(g)=g$ for $t \geqslant s$, the result follows easily. One should note that if $\Psi$ fails the $\Delta_{2}$ condition, one cannot in general take $g$ such that $\|f-g\|_{\Psi}<\|f\|_{\Psi}$.

We have proved that condition $F V_{\Phi}$ implies convergence of $L^{\Psi}$-bounded martingales. We now suppose that $L^{\Psi} \neq L^{1}$, and start to prove the converse. Suppose that condition $F V_{\Phi}$ fails. Then there is an adapted family $\left(A_{t}\right)$, an $\varepsilon>0$, such that for each family $\left(\xi_{t}\right)$ of functions that satisfy the condition

(6) $\xi_{t} \geqslant 0, \xi_{t}=0$ outside $A_{t}, \xi_{t}$ is $\Sigma_{t}$-measurable, $\xi_{t}$ is bounded, only finitely many functions $\xi_{t}$ are nonzero, and $\int \sum \xi_{t} d P \geqslant P(A)-\varepsilon$;

then we have $\left\|\Sigma \xi_{t}-1 \wedge \Sigma \xi_{t}\right\|_{\Phi} \geqslant \varepsilon$. We consider the following three subsets of $L^{\Phi}$.

$$
\begin{aligned}
& C_{1}=\left\{\xi=\Sigma \xi_{t} ;\left(\xi_{t}\right) \text { satisfies }(6)\right\}, \\
& C_{2}=\{\xi ; \xi \leqslant 1\}, \quad C_{3}=\left\{\xi ;\|\xi\|_{\Phi}<\varepsilon\right\} .
\end{aligned}
$$

These sets are convex. Since $C_{3}$ is open, $C_{2}+C_{3}$ is open. Also, $C_{1} \cap\left(C_{2}+C_{3}\right)$ is empty. The theorem of Hahn-Banach implies that there is $h \in\left(L^{\Phi}\right)^{*}$ such that $h \geqslant 1$ on $C_{1}$ and $h<1$ on $C_{2}+C_{3}$. Since $h<1$ on $C_{2}$, we have $h \geqslant 0$.

We note that, for a set $A$, if $P(A) \leqslant 1 / \Phi(a)$, we have $\int \Phi\left(a 1_{A}\right) d P \leqslant 1$, so $\left\|1_{A}\right\|_{\Phi} \leqslant 1 / a$. At this point we use that $\Phi$ is always finite, that is $L^{\Phi} \neq L^{\infty}$.) It follows that $h\left(1_{A}\right) \rightarrow 0$ when $P(A) \rightarrow 0$, so the finitely additive measure $m$ on $\Sigma$ given by $m(A)=h\left(1_{A}\right)$ is in fact absolutely continuous with respect to $P$. It follows that there is $f \in L^{1}, f \geqslant 0$, such that, for $g \in L^{\infty}$, we have $h(g)=E(f g)$.

Let $g \in L^{\Phi}, g>0$, with $\|g\|_{\Phi} \leqslant \varepsilon$. For $a>0$, let $g_{a}$ be the truncation of $g$ at $a$. Since $g_{a} \in L^{\infty}$ and $g_{a} \in C_{3}$, we get

$$
E\left(f g_{a}\right)=h\left(g_{a}\right) \leqslant 1 .
$$

Letting $a \rightarrow \infty$, Fatou's theorem shows that $E(f g) \leqslant 1$. This implies $\|f\|_{\Psi} \leqslant 1 / \varepsilon$, so $f \in L^{\Psi}$. Moreover, $E(f)=h(1) \leqslant 1$, since $h<1$ on $C_{2}$.

For $t \in \Sigma$, and a function $\xi_{t}$ that satisfies $\xi_{t} \geqslant 0, \xi_{t}=0$ outside $A_{t}, E\left(\xi_{t}\right)=$ $P\left(A^{*}\right)-\varepsilon, \xi_{t}$ is $\Sigma_{t}$-measurable, bounded, we have $\xi_{t} \in C_{1}$, so $E\left(\xi_{t} E^{t}(f)\right)=E\left(f \xi_{t}\right)$ $=h\left(\xi_{t}\right) \geqslant 1$. It follows that $E^{t}(f) \geqslant 1 /\left(P\left(A^{*}\right)-\varepsilon\right)$ on $A_{t}$. If $X_{t}=E^{t}(f)$, we then have

$$
P\left(X^{*} \geqslant 1 /\left(P\left(A^{*}\right)-\varepsilon\right)\right) \geqslant P\left(A^{*}\right) .
$$

Since $\|f\|_{1} \leqslant 1$, we also have

$$
P\left(X_{*} \geqslant 1 /\left(P\left(A^{*}\right)-\varepsilon\right)\right) \leqslant P\left(A^{*}\right)-\varepsilon .
$$

This shows that $X^{*} \neq X_{*}$, and hence $\left(X_{t}\right)$ does not converge essentially. The proof of Theorem 6 is complete.

3. Proof of Theorem 7. The core of the proof will be a random choice argument. It relies on the following standard inequality:

Lemma 25. Let $Y$ be a Poisson random variable with $E(Y)=\mu$. Then for $u \geqslant 0$,

$$
P(Y \geqslant \mu(1+u)) \leqslant \exp (-u \theta(u) \mu)
$$

where $\theta(u)=(1+1 / u) \log (1+u)-1$. 
Proof. Use $P(Y \geqslant t) \leqslant E(\exp (h Y-h t))$ for $h \geqslant 0$, and minimize over $h$.

Before we embark on the proof of Theorem 7 , we settle a purely technical point.

Lemma 26. Assume that condition $F V_{\Phi}$ holds. Then for each adapted family $\left(A_{t}\right)$ of sets, each $\gamma>0$, each $\varepsilon>0$, there exists a family $\left(\xi_{t}\right)$ of functions, $\xi_{t} \geqslant 0, \xi_{t}=0$ outside $A_{t}, \xi_{t}$ is bounded, $\Sigma_{t}$-measurable, only finitely many functions $\xi_{t}$ are nonzero, such that if we set $\xi=\sum \xi_{t}, \xi^{\prime \prime}=\xi \wedge 1, \xi^{\prime}=\xi-\xi^{\prime \prime}$, we have

$$
E\left(\xi^{\prime \prime}\right) \geqslant P\left(A^{*}\right)-\varepsilon, \quad \int \Phi\left(\xi^{\prime} / \gamma\right) d P \leqslant \varepsilon .
$$

Proof. Let $u_{0} \geqslant 0$ and $\alpha>0$ be such that $\Phi(u) \geqslant \alpha u$ for $u \geqslant u_{0}$. For any function $f \geqslant 0$ we have

$$
\int_{\left\{f \geqslant \gamma u_{0}\right\}} f d P \leqslant \frac{\gamma}{\alpha} \int \Phi(f / \gamma) d P .
$$

so

$$
\int f d P \leqslant \gamma u_{0}+\frac{\gamma}{\alpha} \int \Phi(f / \gamma) d P .
$$

Let $0<\varepsilon<1$. There is $\gamma_{0}>0$ such that, for $\gamma \leqslant \gamma_{0}$, we have $E(f) \leqslant \varepsilon / 2$ whenever $\int \Phi(f / \gamma) d P \leqslant 1$. Let $\gamma \leqslant \gamma_{0}$. Condition $F V_{\Phi}$ shows that there exists a family $\left(\xi_{t}\right)$ of functions, $\xi_{t} \geqslant 0, \xi_{t}=0$ outside $A_{t}$, $\xi_{t}$ is bounded, $\Sigma_{t}$-measurable, only finitely many functions $\xi_{t}$ are nonzero, and $E\left(\sum \xi_{t}\right) \geqslant P\left(A^{*}\right)-\varepsilon / 2,\left\|\xi^{\prime}\right\|_{\Phi} \leqslant \varepsilon \gamma$, where $\xi=\Sigma \xi_{t}$, $\xi^{\prime \prime}=\xi \wedge 1, \xi^{\prime}=\xi-\xi^{\prime \prime}$. Since $\int \Phi\left(\xi^{\prime} / \varepsilon \gamma\right) d P \leqslant 1$, we have $\int \Phi\left(\xi^{\prime} / \gamma\right) d P \leqslant \varepsilon \leqslant 1$. This implies $E\left(\xi^{\prime}\right) \leqslant \varepsilon / 2$, so $E\left(\xi^{\prime \prime}\right) \geqslant P\left(A^{*}\right)-\varepsilon$. The proof is complete.

We now start the proof of Theorem 7.

First step. Since $\Phi$ satisfies condition Exp, there is $a>0$ such that $\Phi(u) \leqslant \exp a u$ for $u \geqslant 0$. We fix $\gamma>0, b>0$, and we fix an adapted family $\left(A_{t}\right)$ with $P\left(A^{*}\right)>\gamma$. Let $u_{0}$ be large enough that

$$
e^{2 a b+4} \leqslant 2^{-18} \gamma^{2}\left(\theta\left(u_{0}\right)-a b\right) ; \quad e^{a b} \leqslant 1+u_{0} .
$$

We let $\eta=1 /\left(u_{0}+1\right)$. We note that $\eta$ depends only on $b$ and $\gamma$.

Second step. Let $t_{0} \in J$ with $P(D) \leqslant \frac{8}{7} P\left(A^{*}\right)$, where $D$ is the essential union of the sets $A_{t}$ for $t \geqslant t_{0}$. Let $A_{t}^{\prime}=A_{t}$ for $t \geqslant t_{0}$, and $A_{t}^{\prime}=\varnothing$ otherwise. Then $A^{\prime *}=A^{*}$. By Lemma 26, there is a finite set $I \subset J$, with $t_{0} \leqslant I$, and for $i \in I$ a bounded $\Sigma_{i}$-measurable function $\xi_{i}$, with $\xi_{i} \geqslant 0, \xi_{i}=0$ outside $A_{i}$, and

$$
E\left(\xi^{\prime \prime}\right) \geqslant \frac{6}{7} P\left(A^{*}\right) ; \quad \int \Phi\left(2 b \xi^{\prime}\right) d P \leqslant 2^{-17} \eta^{2} \gamma^{2}
$$

where $\xi=\Sigma \xi_{i}, \quad \xi^{\prime \prime}=\xi \wedge 1, \quad \xi^{\prime}=\xi-\xi^{\prime \prime}$. Since $\xi^{\prime \prime}=0$ outside $D$, and $E\left(\xi^{\prime \prime}\right)$ $\geqslant \frac{3}{4} P(D)$, we have $P\left(F_{0}\right) \geqslant \frac{1}{2} P(D)$, where $F_{0}=\left\{\xi^{\prime \prime} \geqslant \frac{1}{2}\right\}$. In particular, $P\left(F_{1}\right)$ $\geqslant \frac{3}{8} P(D) \geqslant \frac{3}{8} P\left(A^{*}\right)$, where $F_{1}=A^{*} \cap F_{0}$. Note also that we can assume $0 \leqslant \xi_{i} \leqslant 1$ for each $i$.

For $i \in I, k \geqslant 1$, let $B_{i, k}=\left\{2^{-k}<\xi_{i} \leqslant 2^{-k+1}\right\}$. We note that

$$
\sum_{i, k} 2^{-k} 1_{B_{i, k}}<\xi \leqslant \sum_{i, k} 2^{-k+1} 1_{B_{i, k}} .
$$


We fix $k_{0}$ such that if

$$
F=F_{1} \cap\left\{\sum_{i \in I, k \leqslant k_{0}} 2^{-k} 1_{B_{i, k}} \geqslant 2^{-3}\right\},
$$

then $P(F) \geqslant \frac{1}{4} P\left(A^{*}\right) \geqslant \gamma / 4$.

Third step. We now consider a standard probability space $\left(\Omega^{\prime}, \Xi, Q\right)$. On $\Omega^{\prime}$, we consider independent Poisson random variables $\varepsilon_{i, k}$, for $i \in I, k \leqslant k_{0}$, such that $E\left(\varepsilon_{i, k}\right)=\eta 2^{-k}$.

On the product $\Omega \times \Omega^{\prime}$, we consider the random variable $Z\left(\omega, \omega^{\prime}\right)$ given by

$$
Z\left(\omega, \omega^{\prime}\right)=\sum_{k \leqslant k_{0} ; i \in I} \varepsilon_{i, k}\left(\omega^{\prime}\right) 1_{B_{i, k}}(\omega) .
$$

We shall bound the integral

$$
\mathscr{I}=\int_{\{Z>1\}} \Phi(b Z) d P d Q .
$$

We fix $\omega \in \Omega$. Let $\mu(\omega)=\int Z\left(\omega, \omega^{\prime}\right) d Q\left(\omega^{\prime}\right)$. If $L=\left\{i, k ; \omega \in B_{i, k}\right\}$, we have $\mu(\omega)=\eta \sum_{(i, k) \in L} 2^{-k}$, so in particular $\mu(\omega) \leqslant \eta \xi(\omega)$; that is, $\left(1+u_{0}\right) \mu(\omega) \leqslant \xi(\omega)$.

Let us first suppose that $2 \leqslant\left(1+u_{0}\right) \mu(\omega)$. Since $\xi(\omega) \geqslant 2$, we have

$$
\begin{aligned}
\int_{\left\{Z \leqslant \mu(\omega)\left(1+u_{0}\right)\right\}} \Phi( & \left.b Z\left(\omega^{\prime}, \omega\right)\right) d Q\left(\omega^{\prime}\right) \leqslant \Phi\left(b\left(1+u_{0}\right) \mu(\omega)\right) \\
& \leqslant \Phi(b \xi(\omega)) \leqslant \Phi(2 b(\xi(\omega)-1)) \leqslant \Phi\left(2 b \xi^{\prime}(\omega)\right) .
\end{aligned}
$$

Now $\omega^{\prime} \rightarrow Z\left(\omega, \omega^{\prime}\right)$ is Poisson of parameter $\mu(\omega)$, so, since $\theta$ is increasing, we have from Lemma 26, for $u \geqslant u_{0}$,

$$
Q(\{Z>\mu(\omega)(1+u)\}) \leqslant \exp \left(-\theta\left(u_{0}\right) u \mu(\omega)\right)
$$

so, with some elementary computations, we get, using (7).

$$
\begin{aligned}
\int_{\left\{Z \geqslant \mu(\omega)\left(1+u_{0}\right)\right\}} \Phi\left(b Z\left(\omega^{\prime}, \omega\right)\right) d Q & \left(\omega^{\prime}\right)=\int_{\mu(\omega)\left(1+u_{0}\right)}^{\infty} \Phi(b u) Q(Z \geqslant u) d u \\
& \leqslant \int_{\mu(\omega)\left(1+u_{0}\right)}^{\infty} \exp \left(a b u-\theta\left(u_{0}\right)(u-\mu(\omega))\right) d u \\
& \leqslant\left(\theta\left(u_{0}\right)-a b\right)^{-1}\left(\eta e^{a b+1}\right)^{\mu(\omega)\left(1+u_{0}\right)} \\
& \leqslant \eta^{2}\left(\theta\left(u_{0}\right)-a b\right)^{-1} e^{2 a b+2} \leqslant 2^{-18} \eta^{2} \gamma^{2} .
\end{aligned}
$$

Suppose now that $\left(1+u_{0}\right) \mu(\omega) \leqslant 2$. Then $Z>1$ forces $Z \geqslant 2$, so $\{Z>1\} \subset$ $\left\{Z \geqslant\left(1+u_{0}\right) \mu(\omega)\right\}$. The second part of the computation above shows that

$$
\begin{aligned}
\int_{\{Z>1\}} \Phi\left(b Z\left(\omega^{\prime}, \omega\right)\right) d Q\left(\omega^{\prime}\right) & \leqslant \int_{2}^{\infty} \exp \left(a b u-\theta\left(u_{0}\right)(u-\mu(\omega))\right) d u \\
& \leqslant\left(\theta\left(u_{0}\right)-a b\right)^{-1} \exp \left(2 a b+\theta\left(u_{0}\right) \mu(\omega)-2 \theta\left(u_{0}\right)\right) \\
& \leqslant \eta^{2}\left(\theta\left(u_{0}\right)-a b\right) e^{2 a b+4} \leqslant 2^{-18} \eta^{2} \gamma^{2}
\end{aligned}
$$

since $\mu(\omega) \theta\left(u_{0}\right) \leqslant 2 \eta \theta\left(u_{0}\right) \leqslant 2$ and $2 \theta\left(u_{0}\right) \geqslant 2 \log \left(1+u_{0}\right)-2$.

Integrating over $\Omega$ and using (8) we get $\mathscr{I} \leqslant 2^{-16} \eta^{2} \gamma^{2}$. 
Let

$$
U=\left\{\omega^{\prime} ; \int_{\{Z>1\}} \Phi\left(b Z\left(\omega^{\prime}, \omega\right)\right) d P(\omega) \leqslant 2^{-7} \eta \gamma\right\} .
$$

From Fubini's theorem and the majoration $\mathscr{I} \leqslant 2^{-16} \eta^{2} \gamma^{2}$ we get $Q(U) \geqslant 1-2^{-9} \eta \gamma$. Fourth step. Again we fix $\theta$. We have

$$
Q\left(\left\{\omega^{\prime} ; Z\left(\omega, \omega^{\prime}\right) \geqslant 1\right\}\right)=1-\exp (-\mu(\omega)) .
$$

Note that $1-e^{-x} \geqslant x / 2$ for $0 \leqslant x \leqslant 1$. For $\omega \in F_{1}$ we have $\mu(\omega) \geqslant \eta 2^{-3}$ so $1-\exp (-\mu(\omega)) \geqslant \eta 2^{-4}$, so we get

$$
P \otimes Q\left(A^{*} \times \Omega^{\prime} \cap\{Z \geqslant 1\}\right) \geqslant 2^{-7} \eta \gamma .
$$

Let

$$
V=\left\{\omega^{\prime} ; P\left(A^{*} \cap\left\{\omega ; Z\left(\omega, \omega^{\prime}\right) \geqslant 1\right\}\right) \geqslant 2^{-8} \eta \gamma\right\} .
$$

We then have $Q(V) \geqslant 2^{-8} \eta \gamma$. This shows that $U \cap V \neq \varnothing$. We fix $\omega^{\prime} \in U \cap V$.

Define $\tau \in I M$ by $\{\tau=t\}=\varnothing$ for $t \notin I$, and for $i \in I$ by

$$
\{\tau=i\}=\bigcup B_{i, k}
$$

where the union is taken over all the $k \leqslant k_{0}$ for which $\varepsilon_{i, k}\left(\omega^{\prime}\right)=1$. Since $B_{i, k} \subset A_{i}$, we have $A(\tau) \supset B_{i, k}$ whenever $\varepsilon_{i, k}\left(\omega^{\prime}\right)=1$, so $\left\{\omega: Z\left(\omega, \omega^{\prime}\right) \geqslant 1\right\} \subset A(\tau)$. Since $\omega^{\prime} \in V$, we have $P\left(A(\tau) \cap A^{*}\right) \geqslant 2^{-4} \eta \gamma$. We have $S_{\tau}(\omega) \leqslant Z\left(\omega, \omega^{\prime}\right)$ and since $\omega^{\prime} \in U$, we get

$$
\int \Phi\left(b e_{\tau}\right) d P \leqslant \int_{\left\{S_{\tau}>1\right\}} \Phi\left(b S_{\tau}\right) d P \leqslant 2^{-4} \eta \gamma
$$

Fifth step. Now let $\varepsilon>0$. In the above construction, let $\gamma=\varepsilon / 2, b=2 / \varepsilon$. We have shown that there is $\delta=2^{-8} \eta \varepsilon$, depending only on $\varepsilon$, such that whenever $\left(A_{t}\right)$ is an adapted family with $P\left(A^{*}\right)>\varepsilon$, there is $\tau \in I M$ with $P\left(A(\tau) \cap A^{*}\right) \geqslant \delta$ and $\int \Phi\left(2 e_{\tau} / \varepsilon\right) d P \leqslant \delta$. The conclusion of the proof follows the standard exhausting procedure. Let us fix an adapted family $\left(A_{t}\right)$ with $P\left(A^{*}\right)>\varepsilon$. By induction over the integer $k$, we construct $\tau_{k} \in I M$ such that $P\left(A\left(\tau_{k}\right) \cap A^{*}\right) \geqslant \delta k$ and $\int \Phi\left(2 e_{\tau_{k}} / \varepsilon\right) d P$ $\leqslant k \delta$. The induction continues as long as $P\left(A^{*} \backslash A\left(\tau_{k}\right)\right)>\varepsilon$, so it finishes in at most $1 / \delta$ steps. We have just proved the possibility of the first step. Assume now that $\tau_{k}$ has been constructed, and that $P\left(A^{*} \backslash A\left(\tau_{k}\right)\right)>\varepsilon$. We can assume $\left\{\tau_{k}=t\right\}$ $\subset A_{t}$ for each $t$. Let $t_{0} \in I$ be such that $\tau_{k} \leqslant t_{0}$. For $t \geqslant t_{0}$, let $A_{t}^{\prime}=A_{t} \backslash A\left(\tau_{k}\right)$, and let $A_{t}^{\prime}=\varnothing$ otherwise. We have $P\left(A^{\prime *}\right)>\varepsilon$, so there is $\tau \in I M$ with $P\left(A^{\prime *} \cap A(\tau)\right)$ $\geqslant \delta$ and $\int \Phi\left(2 e_{\tau} / \varepsilon\right) d P \leqslant \delta$. We define $\left\{\tau_{k+1}=t\right\}=\left\{\tau_{k}=t\right\}$ for $t \leqslant t_{0},\left\{\tau_{k+1}=\right.$ $t\}=\{\tau=t\} \backslash A\left(\tau_{k}\right)$ for $t \geqslant t_{0}$ and $\left\{\tau_{k}=t\right\}=\varnothing$ otherwise, and it is straightforward to check that $\tau_{k+1}$ satisfies the requirements. When the induction stops, we have constructed $\tau^{\prime} \in I M$ with $P\left(A^{*} \backslash A\left(\tau^{\prime}\right)\right)<\varepsilon$ and

$$
\int \Phi\left(2 e_{\tau^{\prime}} / \varepsilon\right) d P \leqslant P\left(A^{*} \cap A\left(\tau^{\prime}\right)\right) \leqslant 1,
$$

so $\left\|2 e_{\tau^{\prime}} / \varepsilon\right\|_{\Phi} \leqslant 2$ and $\left\|e_{\tau^{\prime}}\right\|_{\Phi} \leqslant \varepsilon$. The proof is complete.

4. Proof of Theorem 8. We start a series of lemmas that will culminate in the proof of the necessity of condition $D_{\Phi}^{\prime}$ when $J$ contains a countable cofinal set. For clarity we suppose in this paragraph that $L^{\Phi} \neq L^{\infty}$. The case of $L^{1}$-bounded martingales will be investigated in the next paragraph. 
LEMMA 27. Let $N_{1}, N_{2}$ be two lattice norms on $L^{\Phi}$, that are equivalent to the norm $\|\cdot\|_{\Phi}$. Let $I \subset J$ be a finite set, and for $i \in I$ let $A_{i}$ be a $\Sigma_{i}$-measurable set. Assume that for each family $\left(\xi_{i}\right)_{i \in I}$ of bounded functions, with $\xi_{i} \geqslant 0$, each $\xi_{i}$ is $\Sigma_{i}$-measurable, $\xi_{i}=0$ outside $A_{i}$, and $\left\|\sum_{i \in I} \xi_{i}\right\|_{1}=1$, we have either $N_{1}\left(\Sigma \xi_{i}\right) \geqslant 1$ or $N_{2}\left(\sum \xi_{i}\right) \geqslant 1$. Then there exist two functions $f_{1}, f_{2} \in L^{\Psi}$ such that for $j=1,2$ we have

$$
\forall g \in L^{\Phi}, \quad N_{j}(g) \leqslant 1 \Rightarrow E\left(\left|f_{j} g\right|\right) \leqslant 1
$$

and that for $i$ in I we have

$$
A_{i} \subset\left\{E^{i}\left(f_{1}+f_{2}\right) \geqslant 1\right\} .
$$

Proof. Consider the set $C_{1}$ of functions $\xi=\sum_{i \in I} \xi_{i}$, where the functions $\xi_{i}$ satisfy the conditions of the lemma. It is a convex set. Let $C_{2}=\left\{g \in L^{\Phi}, N_{1}(g)<1, N_{2}(g)\right.$ $<1\}$. It is a convex open set of $L^{\Phi}$, and $C_{1} \cap C_{2}=\varnothing$ by hypothesis. The theorem of Hahn-Banach gives an $h$ in the dual $G$ of $L^{\Phi}$ with $h<1$ on $C_{2}$ and $h \geqslant 1$ on $C_{1}$. For $j=1,2$, let

$$
M_{j}=\left\{l \in G:|l(g)| \leqslant 1 \text { for } g \in L^{\Phi}, N_{j}(g) \leqslant 1\right\} .
$$

Let

$$
M=\left\{l \in G:|l(g)| \leqslant 1 \text { for } g \in L^{\Phi}, N_{1}(g) \leqslant 1, N_{2}(g) \leqslant 1\right\} .
$$

A straightforward use of the theorem of Hahn-Banach shows that $M$ is the convex hull of $M_{1}$ and $M_{2}$; that is, we can write $h=\lambda_{1} h_{1}+\lambda_{2} h_{2}$ where $\lambda_{1}, \lambda_{2} \geqslant 0$, $\lambda_{1}+\lambda_{2}=1, h_{1} \in M_{1}, h_{2} \in M_{2}$.

For $i \in I$, consider the restriction of $h_{1}$ (resp. $h_{2}$ ) to $L^{\infty}\left(\Omega, \Sigma_{i}, P\right)$. Since $L^{\Phi} \neq L^{\infty}$, we have shown in the proof of Theorem 6 that for $j=1,2$ there is $f_{j}^{\prime} \in L^{1}$ such that $h_{j}(g)=E\left(f_{j}^{\prime} g\right)$ for $g \in L^{\infty}$. Let $f_{j}=\left|f_{j}^{\prime}\right|$. If $g \in L^{\Phi}$ with $N_{j}(g) \leqslant 1, g \geqslant 0$, for $a>0$ its truncation $g_{a}$ at $a$ satisfies $N_{j}\left(g_{a}\right) \leqslant 1$, so if $g_{a}^{\prime}=g_{a} \operatorname{sign} f_{j}^{\prime}$ we have

$$
E\left(f_{j} g_{a}\right)=E\left(f_{j}^{\prime} g_{a}^{\prime}\right)=h\left(g_{a}^{\prime}\right) \leqslant 1 \text {. }
$$

Letting $a \rightarrow \infty$, Fatou's lemma gives $E\left(f_{j} g\right) \leqslant 1$. For $g \in C_{1}$, we have

$$
1 \leqslant h(g)=\lambda_{1} E\left(f_{1}^{\prime} g\right)+\lambda_{2} E\left(f_{2}^{\prime} g\right) \leqslant E\left(\left(f_{1}+f_{2}\right) g\right)
$$

and this shows that $A_{i} \subset\left\{E^{i}\left(f_{1}+f_{2}\right) \geqslant 1\right\}$, and concludes the proof.

Lemma 28. Assume that $J$ contains a countable cofinal set. Let $\varepsilon, \delta>0$ be fixed. Assume that for each $s \in J$ and each $\eta \geqslant 0$, there is a finite set $I \subset J, s \leqslant I$ and $g \in L^{\Psi}$ with $\int \Psi(g \delta) d P<\eta$, and $P\left(\bigcup_{i \in I} B_{i}\right) \geqslant \varepsilon / 2$ where $B_{i}=\left\{E^{i}(g) \geqslant \frac{1}{2}\right\}$. Then there is $f$ in $L^{\Psi}$ such that the martingale $\left(E^{t}(f)\right)$ fails to converge essentially.

Proof. We first note that, for each $\gamma>0$, there is $\eta>0$ such that $\int \Psi(g \delta) d P \leqslant \eta$ implies $\|g\|_{1} \leqslant \gamma$. Indeed, there is $\alpha, u_{0}>0$ such that $\Psi(u)-\Psi\left(u_{0}\right) \geqslant \alpha\left(u-u_{0}\right)$ for $u \geqslant u_{0}$, and hence

$$
\int_{\left\{g \delta>u_{0}\right\}} g d P \leqslant \delta^{-1}\left(\alpha^{-1} \int \Psi(\delta g) d P+P\left(\left\{g \delta>u_{0}\right\}\right)\right) .
$$

Moreover, since we assume (5), we have $\Psi(u)>0$ for $u>0$, so $g$ goes to zero in measure when $\int \Psi(\delta g) d P$ goes to zero; so the claim follows. 
Let $\left(s_{k}\right)$ be a countable cofinal set of $J$. By induction, we construct finite sets $I_{k}$, with $s_{k} \leqslant I_{k}$, sets $\left(A_{k}\right)_{i \in I_{k}}$, a decreasing sequence of numbers $a_{k} \geqslant 0$, such that if we set $p_{k}=$ card $I_{k}$, the following conditions hold:

$$
\begin{aligned}
& a_{k}<a_{k-1} / 2 \text {, and if } B \in \Sigma, P(B)<a_{k}, \text { then for } j \leqslant k \text { we have } \\
& \int_{B} f_{j} d P \leqslant 2^{-j-3} p_{j}^{-1} .
\end{aligned}
$$

$$
\begin{gathered}
\int \Psi\left(\delta f_{k}\right) d P \leqslant 2^{-k} ; \quad\left\|f_{k}\right\|_{1} \leqslant \operatorname{Inf}\left(2^{-k-5} \varepsilon, a_{k-1} / 8\right) . \\
P\left(\bigcup_{i \in I_{k}} A_{i}\right) \geqslant \varepsilon / 2 \quad \text { where for } i \in I_{k}, A_{i}=\left\{E^{i}\left(f_{k}\right) \geqslant \frac{1}{2}\right\} .
\end{gathered}
$$

Let $B_{k}=\left\{f_{k} \geqslant \frac{1}{4}\right\}$. Since $\left\|f_{k}\right\|_{1} \leqslant a_{k-1} / 8$, we have $P\left(B_{k}\right) \leqslant a_{k-1} / 2$. Let $h_{k}=$ $f_{k} I_{B_{k}}$. Since $E^{i}\left(f_{k}-h_{k}\right) \leqslant \frac{1}{4}$ for each $i$ we have $A_{i} \subset\left\{E^{i}\left(h_{k}\right) \geqslant \frac{1}{4}\right\}$. Let $C_{k}=$ $\bigcup_{j>k} B_{j}$, and $g_{k}=h_{k} 1_{X / C_{k}}$. We note that the functions $g_{k}$ live on disjoint sets. Also, since $P\left(C_{k}\right) \leqslant a_{k}$, we have $\left\|h_{k}-g_{k}\right\|_{1} \leqslant 2^{-k-3} / p_{k}$. So if $A_{i}^{\prime}=\left\{E^{i}\left(g_{k}\right) \geqslant \frac{1}{8}\right\}$ then $P\left(A_{i} \backslash A_{i}^{\prime}\right) \leqslant 2^{-k} / p_{k}$. In particular, we get

$$
P\left(\bigcup_{i \in I_{k}}\left\{E^{i}\left(g_{k}\right) \geqslant \frac{1}{8}\right\}\right) \geqslant \varepsilon / 2-2^{-k} .
$$

Let $f=\sum_{k \geqslant 1} g_{k}$. Since $g_{k} \leqslant f_{k}$, we have $\int \Psi\left(\delta^{-1} g_{k}\right) d P \leqslant 2^{-k}$. Since the functions $g_{k}$ live on disjoint sets, we have $\int \Psi\left(\delta^{-1} f\right) d P \leqslant 1$. In particular, $f \in L^{\Psi}$. Since $g_{k} \leqslant f_{k}$, we have $\left\|g_{k}\right\|_{1} \leqslant 2^{-k-5} \varepsilon$, so $\|f\|_{1} \leqslant 2^{-5} \varepsilon$. Let $X_{t}=E^{t}(f)$. It follows from (12) that $P\left(X^{*} \geqslant \frac{1}{8}\right) \geqslant \varepsilon / 2$. On the other hand, we have $\int X_{*} d P \leqslant \int f d P \leqslant 2^{-5} \varepsilon$, so $P\left(X_{*} \geqslant \frac{1}{8}\right) \leqslant \varepsilon / 4$. This shows that $\left(X_{t}\right)$ does not converge essentially, and concludes the proof.

LEMMA 29. Suppose that $J$ contains a countable cofinite set, and that for each $f \in L^{\Psi}$, the martingale $E^{t}(f)$ converges essentially. Let $\varepsilon>0$. Then there is $\eta_{0}$, depending only on $\varepsilon$, such that, for each $\delta>0$, there are $\eta>0$ and $s$ in $J$, depending only on $\varepsilon$ and $\delta$, such that, for each finite set $I$ with $s \leqslant I$, and each family $\left(A_{i}\right)_{i \in I}$, where $A_{i}$ is a $\Sigma_{i}$-measurable set, such that $P\left(\cup A_{i}\right) \geqslant \varepsilon$, there is a family $\left(\xi_{i}\right)$ of functions, where $\xi_{i}$ is $\Sigma_{i}$-measurable, $\xi_{i} \geqslant 0, \xi_{i}=0$ outside $A_{i},\left\|\Sigma \xi_{i}\right\|_{1}=1$ and

$$
\int \Phi\left(\eta_{0} \sum \xi_{i}\right) d P \leqslant \eta_{0}, \quad \int \Phi\left(\eta \sum \xi_{i}\right) d P \leqslant \eta \delta .
$$

Proof. First step. We show that there are $\eta_{0}$ and $s_{0}$ such that, for each finite set $I$ with $s_{0} \leqslant I$, and each family $\left(A_{i}\right)_{i \in I}$ of $\Sigma_{i}$-measurable sets, with $P\left(\cup A_{i}\right) \geqslant \varepsilon / 2$ there is a family $\left(\xi_{i}\right)$ of bounded functions, where $\xi_{i}$ is $\Sigma_{i}$-measurable, $\xi_{i} \geqslant 0, \xi_{i}=0$ outside $A_{i},\left\|\sum \xi_{i}\right\|_{1}=1$ and $\int \Phi\left(4 \eta_{0} \sum \xi_{i}\right) d P \leqslant \eta_{0}$.

Suppose this fails. So, for each $\eta_{0}>0$ and $s_{0} \in J$, there is a finite set $I$ with $s_{0} \leqslant I$, and a family $\left(A_{i}\right)_{i \in I}$ of $\Sigma_{i}$-measurable sets with $P\left(\bigcup A_{i}\right) \geqslant \varepsilon / 2$, such that we cannot find a family $\left(\xi_{i}\right)$ of functions, where $\xi_{i}$ is $\Sigma_{i}$-measurable, $\xi_{i} \geqslant 0, \xi_{i}=0$ outside $A_{i},\left\|\sum \xi_{I}\right\|_{1}=1$ and $\int \Phi\left(4 \eta_{0} \sum \xi_{i}\right) d P \leqslant \eta_{0}$. 
We now use Lemma 27 with $N_{1}(\cdot)=N_{2}(\cdot)=\|\cdot\|_{\Phi^{\prime}}$, where $\Phi^{\prime}(u)=\eta_{0}^{-1} \Phi\left(4 \eta_{0} u\right)$. (The conjugate Young function $\Psi^{\prime}$ is given by $\Psi^{\prime}(u)=\eta_{0}^{-1} \Psi(u / 4)$.) So there is $f \in L^{\Psi^{\prime}}$ such that $E(|f g|) \leqslant 2$ for $\|g\|_{\Phi^{\prime}} \leqslant 1$, and such that $A_{i} \subset\left\{E\left(f^{i}\right) \geqslant 1\right\}$. We see that $\|f\|_{\Psi^{\prime}} \leqslant 2$, so $\int \Psi^{\prime}(f / 2) d P \leqslant 1$, so $\int \Psi(f / 8) d P \leqslant \eta_{0}$. The conclusion follows from Lemma 28 (with $\delta=\frac{1}{8}$ ).

Second step. We finish the proof. Suppose that the conclusion fails. Then there exists $\delta>0$ such that, for each $\eta>0$ and $s \geqslant s_{0}$, there exist a finite set $I$ with $s \leqslant I$ and a family $\left(A_{i}\right)_{i \in I}$ of $\Sigma_{\Gamma}$-measurable sets with $P\left(\bigcup A_{i}\right) \geqslant \varepsilon / 2$, such that for each family $\left(\xi_{i}\right)$ of functions, where $\xi_{i}$ is $\Sigma_{i}$-measurable, $\xi_{i} \geqslant 0, \xi_{i}=0$ outside $A_{i}$, $\left\|\sum \xi_{i}\right\|_{1}=1$, we get either $\int \Phi\left(\eta_{0} \sum \xi_{i}\right) d P \geqslant \eta_{0}$ or $\int \Phi\left(\eta \sum \xi_{i}\right) d P \geqslant \eta \delta$.

We now use Lemma 27 with $N_{1}(\cdot)=\|\cdot\|_{\Phi^{\prime \prime}}, N_{2}(\cdot)=\|\cdot\|_{\Phi}$, where $\Phi^{\prime \prime}(u)=$ $\eta_{0}^{-1} \Phi\left(\eta_{0} u\right), \Phi^{\tilde{}}(u)=(\delta \eta)^{-1} \Phi(\eta u)$. (The conjugate Young functions are given, respectively, by $\Psi^{\prime \prime}(u)=\eta_{0}^{-1} \Psi(u)$ and $\Psi^{\sim}(u)=(\delta \eta)^{-1} \Psi(\delta u)$.) It follows that there are $g_{1}, g_{2}$ with $\left\|g_{1}\right\|_{\Psi^{\prime \prime}} \leqslant 1,\left\|g_{2}\right\|_{\Psi^{-}} \leqslant 1$ such that $A_{i} \subset\left\{E^{i}\left(g_{1}+g_{2}\right) \geqslant 1\right\}$.

Let $C_{i}=\left\{E^{i}\left(g_{1}\right)>\frac{1}{2}\right\}$. Suppose, if possible, that $P\left(\cup C_{i}\right) \geqslant \varepsilon / 2$. Then the first step gives functions $\xi_{i}, \xi_{i} \geqslant 0, \xi_{i}=0$ outside $C_{i}, \xi_{i}$ is $\Sigma_{i}$-measurable, $\left\|\sum \xi_{i}\right\|_{1}=1$, and $\int \Phi\left(4 \eta \sum \xi_{i}\right) d P \leqslant \eta_{0}$, so $\int \Phi^{\prime}\left(4 \sum \xi_{i}\right) d P \leqslant 1$, so $\left\|\sum \xi_{i}\right\|_{\Phi^{\prime}} \leqslant \frac{1}{2}$. It follows that $E\left(g_{1} \sum \xi_{i}\right)$ $\leqslant \frac{1}{2}$. However, since $\xi_{i}$ is $\Sigma_{i}$-measurable,

$$
\begin{aligned}
E\left(g_{1} \sum \xi_{i}\right) & =\sum E\left(g_{1} \xi_{i}\right)=\sum\left(\xi_{i} E^{i}\left(g_{1}\right)\right) \\
& >\frac{1}{2} \sum E\left(\xi_{i}\right) \geqslant \frac{1}{2}
\end{aligned}
$$

since $E^{i}\left(g_{1}\right)>\frac{1}{2}$ when $\xi_{i}>0$. This contradiction proves that $P\left(\cup C_{i}\right) \leqslant \varepsilon / 2$. Let $A_{i}^{\prime}=\left\{E^{i}\left(g_{2}\right) \geqslant \frac{1}{2}\right\}$. Since $P\left(\bigcup A_{i}\right) \geqslant \varepsilon$ we have $P\left(\bigcup A_{i}^{\prime}\right) \geqslant \varepsilon / 2$. Since $\left\|g_{2}\right\|_{\Psi^{-}} \leqslant 1$, we have $\int \Psi\left(\delta g_{2}\right) d P \leqslant \eta \delta$. But Lemma 28 shows this is impossible. This concludes the proof.

LEMMA 30. Suppose that $J$ contains a countable cofinite set, and that $L^{\Psi}$-bounded martingales converge essentially. Let $\varepsilon>0$. Then there is $\eta_{1}$, depending only on $\varepsilon$, such that for each $\delta>0$ there are $\eta>0, s \in J, \gamma>0, b>0$, depending only on $\varepsilon$ and $\delta$, such that, for every finite adapted famly of sets $\left(A_{t}\right)$ with

$$
\varepsilon<P\left(\bigcup_{t \geqslant s} A_{t}\right) ; \quad \sum_{t \geqslant s} P\left(A_{t}\right) \leqslant(1+\gamma) P\left(\bigcup_{t \geqslant s} A_{t}\right),
$$

there is $\tau \in I M$ with $\tau \geqslant s$ such that $P(A(\tau)) \geqslant b$ and

$$
\int \Phi\left(\eta_{1} S_{\tau}\right) d P \leqslant 1 ; \quad \int \Phi\left(\eta S_{\tau}\right) d P \leqslant \delta \eta P(A(t)) .
$$

Proof. First step. Let $\delta>0$, and let $\eta_{0}=\eta_{0}(\varepsilon), \eta_{1}=\eta_{0} / 4$, and $\eta=\eta\left(\varepsilon, \delta 2^{-4}\right)$ be as in Lemma 29. Since we assumed that $\lim \phi(t)=\infty$, there is $l \in \mathbf{N}$ such that for $u \geqslant 2^{l}$ we have $\Phi\left(\eta_{0} u\right) \geqslant 4 \eta_{0} u$. Let $b=2^{-l-5} /(l+2)$, and $\gamma=2^{-l-2}$. Let

$$
C=\left\{\omega ; \exists i \neq j, i, j \geqslant s, x \in A_{i} \cap A_{j}\right\} .
$$

Then (14) implies that $P(C) \leqslant \gamma$. Let $D=\bigcup_{t \geqslant s} A_{t}$.

Second step. Let $\left(\xi_{i}\right)_{i \in I}$ be as in Lemma 29 but with $\delta 2^{-4}$ instead of $\delta$, and let $\xi=\sum \xi_{i}$. We have $\int_{C \cap\left\{\xi \leqslant 2^{\prime}\right\}} \xi d P \leqslant \frac{1}{4}$, and also

$$
\int_{\left\{\xi \geqslant 2^{l}\right\}} \xi d P \leqslant \frac{1}{4} \int \eta_{0}^{-1} \Phi\left(\eta_{0} \xi\right) d P \leqslant \frac{1}{4} .
$$


It follows that $\int_{D \backslash C} \xi d P \geqslant \frac{1}{2}$. For each $-2<k \leqslant l-1$ and $i \in I$, let

$$
A_{i}^{k}=\left\{2^{k}<\xi_{i} \leqslant 2^{k+1}\right\}
$$

so that $A_{i}^{k} \in \Sigma_{i}$. Let $\tau_{k} \in I M$ be given by $\left\{\tau_{k}=i\right\}=A_{i}^{k}$ for $i \in I,\left\{\tau_{k}=i\right\}=\varnothing$ otherwise. The rest of the proof consists of showing that for $\tau$ one can take one of the $\tau_{k}$. We first note that, for each $i$, we have $\sum_{k} 2^{k} 1_{\left\{\tau_{k}=i\right\}}<\xi_{i}$ so we have

$$
\sum_{k} 2^{k} S_{\tau_{k}} \leqslant \xi
$$

Let $H=(D \backslash C) \cap\left\{\xi>\frac{1}{4}\right\}$. Since $\int_{D \backslash C} \xi d P \geqslant \frac{1}{2}$, we have $\int_{H} \xi d P \geqslant \frac{1}{4}$. For $\omega \in$ $H$, there exists a unique $i$ such that $\xi_{i}(\omega)>0$, so we have $\xi_{i}(\omega)>\frac{1}{4}$, so

$$
\xi_{i}(\omega) \leqslant \sum_{k=-2}^{l-1} 2^{k+1} 1_{\left\{\tau_{k}=i\right\}}(\omega)
$$

Summation over $i$ gives $\xi(\omega) \leqslant \sum_{k=-2}^{l-1} 2^{k+1} S_{\tau_{k}}(\omega)$. For $k \in[-2, l-1]$, let $a_{k}=$ $\int_{H} 2^{k+1} S_{\tau_{k}} d P$. Since $\int_{H} \xi D P \geqslant \frac{1}{4}$, w have $\sum_{k} a_{k} \geqslant \frac{1}{4}$. Now from (13), (16) we deduce that

$$
\sum_{k} \int \Phi\left(\eta 2^{k} S_{\tau_{k}}\right) d P \leqslant 2^{-4} \delta \eta
$$

so we have $\sum a_{k} b_{k} \leqslant 2^{-4} \delta \eta$, where $b_{k}=a_{k}^{-1} \int \Phi\left(\eta 2^{k} S_{\tau_{k}}\right) d P$ for $a_{k} \neq 0$, and zero otherwise. Let

$$
L=\left\{k ; b_{k} \geqslant \delta n / 2\right\} .
$$

Then $\sum_{k \in L} a_{k} \leqslant 2^{-3}$, so $\sum_{k \in L} a_{k} \geqslant \frac{1}{8}$. Let

$$
L^{\prime}=\left\{k \in[-2, l-1] ; a_{k} \geqslant 1 / 16(l+2) ; k \notin L\right\} .
$$

We have $\sum_{k \in L^{\prime}} a_{k} \geqslant 2^{-4}$. It follows that there is $k$ with $b_{k} \leqslant \delta \eta / 2$ and $a_{k} \geqslant$ $1 / 16(l+2)$.

Since the sets $A_{i}^{k}$ are disjoint on $H$, and since $\{\tau=i\} \subset A_{i}$ for each $i$, we get

$$
P\left(H \cap A\left(\tau_{k}\right)\right)=\int_{H} S_{\tau_{k}} d P=2^{-k-1} a_{k} \geqslant 2^{-l-5} /(l+2)=b .
$$

Since $b_{k} \leqslant \delta \eta / 2$, we get $2^{k} \int \Phi\left(\eta_{0} S_{\tau_{k}}\right) d P \leqslant \delta \eta a_{k} / 2$, so, since $2^{-k} a_{k} \leqslant 2 P\left(A\left(\tau_{k}\right)\right)$, we get

$$
\int \Phi\left(\eta S_{\tau_{k}}\right) d P \leqslant \delta \eta P\left(A\left(\tau_{k}\right)\right)
$$

Moreover,

$$
\int \Phi\left(\eta_{1} S_{\tau_{k}}\right) d P \leqslant \int \Phi\left(4 \eta_{1} \xi\right) d P \leqslant \int \Phi\left(\eta_{0} \xi\right) d P \leqslant 1 .
$$

The proof is complete.

Lemma 31. Suppose that $J$ contains a countable cofinal set, and that $L^{\Psi}$-bounded martingales converge essentially. Let $\varepsilon>0$. Then there is $\eta_{1}$, depending only on $\varepsilon$, such that, for each $\delta>0$, there are $s_{1} \in J, \gamma_{1}>0, b_{1}>0$, depending only on $\varepsilon$ and $\delta$, such that for every finite adapted family of sets $\left(A_{t}\right)$ which satisfies

$$
\varepsilon<P\left(\bigcup_{t \geqslant s_{1}} A_{t}\right) ; \quad \sum_{t \geqslant s_{1}} P\left(A_{t}\right) \leqslant\left(1+\gamma_{1}\right) P\left(\bigcup_{t \geqslant s_{1}} A_{t}\right),
$$


there is $\tau \in I M$ with $\tau \geqslant s_{1}$ such that

$$
P(A(\tau)) \geqslant b_{1} \text { and } \int \Phi\left(\eta_{1} e_{\tau}\right) d P \leqslant \delta P(A(\tau)) .
$$

Proof. Fix $\varepsilon>0$. Let $\eta_{1}$ be as in Lemma 30. We can assume $24 \eta_{1} \leqslant 1$. Let $\delta>0$. Let $s, \gamma, b, \eta$ be as in Lemma 30. Let $d=\eta / 2 \eta_{1}$. Let $k$ be large enough that $k \geqslant 4 / \eta \delta b d$. Let $\gamma_{1} \leqslant \gamma$ be small enough that $\gamma_{1} \Phi\left(\eta_{1} k\right) \leqslant n \delta d$. Let $b_{1}=d b / 4$. Let $s_{1}=s$. Let $\left(A_{t}\right)$ be as in the statement of the present lemma. From Lemma 30 there exists $\tau^{\prime} \in I M$ such that $P\left(A\left(\tau^{\prime}\right)\right) \geqslant b$ and

$$
\int \Phi\left(\eta_{1} S_{\tau^{\prime}}\right) d P \leqslant 1, \quad \int \Phi\left(\eta S_{\tau^{\prime}}\right) d P \leqslant \delta \eta P\left(A\left(\tau^{\prime}\right)\right) .
$$

We can suppose that $\left\{\tau^{\prime}=t\right\} \subset A_{t}$ for each $t$. Let

$$
I=\left\{i \in J ;\left\{\tau^{\prime}=1\right\} \neq \varnothing\right\} \text {. }
$$

Let

$$
C=\left\{\omega ; \exists i, j \in I, i \neq j, \omega \in\left\{\tau^{\prime}=i\right\} \cap\left\{\tau^{\prime}=j\right\}\right\} .
$$

From (17), we have $P(C) \leqslant \gamma_{1}$. For $i \in I$, let $a_{i}=P\left(\left\{\tau^{\prime}=i\right\} \cap C\right)$. Since we can assume $\gamma_{1}<b / 2$, we have $\sum a_{i} \geqslant P\left(A\left(\tau^{\prime}\right)\right) / 2$.

We shall obtain $\tau$ by a random choice. More precisely, let $Y=\{0,1\}^{I}$, provided with the probability $Q$ that makes the coordinate functions $\varepsilon_{i}$ independent of expectation $d$. For $y \in Y$, let $\tau_{y}$ be given by $\left\{\tau_{y}=i\right\}=\left\{\tau^{\prime}=i\right\}$ if $y(i)=1$, and $\left\{\tau_{y}=i\right\}=\varnothing$ otherwise. We shall show that, with positive probability, $\tau_{y}$ satisfies (18). For $y$ in $Y$, we have

$$
P\left(A\left(\tau_{y}\right)\right) \geqslant \sum \varepsilon_{i}(y) a_{i} \stackrel{\text { def }}{=} R(y) .
$$

We have $E(R)=d \sum a_{i}$. Let $U=\{y ; R(y)>E(R) / 2\}$. Then

$$
E(R) / 2 \leqslant \int_{U} R d Q \leqslant Q(U)^{1 / 2}\left(E\left(R^{2}\right)\right)^{1 / 2} .
$$

Since $E\left(R^{2}\right) \leqslant d\left(\sum a_{i}\right)^{2}$, we get $Q(U) \geqslant d / 4$. And for $y \in U$, we have

$$
P\left(A\left(\tau_{y}\right)\right)>d \sum a_{i} / 2 \geqslant d P\left(A\left(\tau^{\prime}\right)\right) / 4 \geqslant b d / 4=b_{1} .
$$

Let $D=\left\{S_{\tau^{\prime}} \leqslant k\right\}$. Since $e_{\tau^{\prime}}=0$ outside $C$, we get, by the choice of $\gamma_{1}$,

$$
\int_{D} \Phi\left(\eta_{1} e_{\tau^{\prime}}\right) d P \leqslant \gamma_{1} \Phi\left(\eta_{1} k\right) \leqslant \eta \delta b .
$$

Let $\omega \notin D$. Let $V_{\omega}=\left\{y \in Q ; \sum \varepsilon_{i}(y) \geqslant 2 d S_{\tau^{\prime}}(\omega)\right\}$ where the summation is over the indexes $i$ for which $\omega \in\left\{\tau^{\prime}=i\right\}$. For $y \notin V_{\omega}$, we have $\Phi\left(\eta_{1} e_{\tau_{y}}\right) \leqslant \Phi\left(\eta S_{\tau^{\prime}}(\omega)\right)$. For $y \in V_{\omega}$, we have $\Phi\left(\eta_{1} e_{\tau_{v}}\right) \leqslant \Phi\left(\eta_{1} S_{\tau^{\prime}}(\omega)\right)$. Let

$$
\theta(\omega, y)=\sup \left(\Phi\left(\eta_{1} e_{\tau_{y}}(\omega)\right), \Phi\left(\eta S_{\tau^{\prime}}(\omega)\right)\right)-\Phi\left(\eta S_{\tau^{\prime}}(\omega)\right)
$$

We have shown that

$$
\int \theta(\omega, y) d Q(y) \leqslant Q\left(V_{\omega}\right) \Phi\left(\eta_{1} S_{\tau^{\prime}}(\omega)\right)
$$


The choice of $k$ implies that $Q\left(V_{\omega}\right)<\eta \delta d b / 4$. Integrating over $C \backslash D$ and using (19) gives

$$
\int_{C \backslash D} \theta(\omega, y) d Q(y) d P(\omega)<\eta \delta d b / 4
$$

If $V=\left\{y \in Q ; \int_{D} \theta(\omega, y) d P(\omega) \leqslant \eta \delta b\right\}$, then $Q(V)>1-d / 4$. For $y \in V$, we have

$$
\begin{aligned}
\int \theta\left(\eta_{1} e_{\tau_{y}}\right) d P & =\int_{C} \Phi\left(\eta_{1} e_{\tau_{y}}\right) d P \\
& \leqslant \int_{D} \Phi\left(\eta_{1} e_{\tau^{\prime}}\right) d P+\int_{C \backslash D} \Phi\left(\eta S_{\tau^{\prime}}\right) d P+\int_{C \backslash D} \theta(\omega, y) d P(\omega) \\
& \leqslant 2 \eta \delta b+\eta \delta P\left(A\left(\tau^{\prime}\right)\right) \leqslant 3 \eta S P\left(A\left(\tau^{\prime}\right)\right) .
\end{aligned}
$$

We have $Q(U \cap V)>0$. And for $y \in U \cap V$, we have

$$
b_{1} \leqslant P\left(A\left(\tau^{\prime}\right)\right) \leqslant(4 / d) P\left(A\left(\tau_{y}\right)\right)=\left(8 \eta_{1} / \eta\right) P(A(\tau)),
$$

so

$$
\int \Phi\left(\eta_{1} e_{\tau_{y}}\right) d P \leqslant 24 \eta_{1} \delta P\left(A\left(\tau_{y}\right)\right) \leqslant \delta P\left(A\left(\tau_{y}\right)\right) .
$$

The proof is complete.

LEMMA 32. Suppose that J contains a countable cofinite set, and that $L^{\Psi}$-bounded martingales converge essentially. Then given $\varepsilon>0$, there exists $\eta_{1}=\eta_{1}(\varepsilon)$, such that for $\delta>0$, there is $s_{2} \in J, \gamma_{2}>0, b_{2}>0$ (depending only on $\varepsilon$ and $\delta$ ) and a set $T \subset \Sigma$ with $P(T) \geqslant 1-\varepsilon-\delta$ such that, for every finite adapted family of sets $\left(A_{t}\right)$ which satisfy

$$
\begin{gathered}
P\left(\bigcup_{t \geqslant s_{2}} A_{t} \cap T\right) \geqslant \delta, \\
\sum_{t \geqslant s_{2}} P\left(A_{t}\right) \leqslant\left(1+\gamma_{2}\right) P\left(\bigcup_{t \geqslant s_{2}} A_{t}\right),
\end{gathered}
$$

there is $\tau \in I M$ with $\tau \geqslant s_{2}$ such that

$$
P(A(\tau)) \geqslant b_{2} \text { and } \int \Phi\left(\eta_{1} e_{\tau}\right) d P \leqslant \delta b_{2} .
$$

Proof. Let $\eta_{1}$ be as in Lemma 31 and let $\delta^{\prime} \leqslant \delta$. For an adapted family $A=\left(A_{t}\right)$, write

$$
\begin{aligned}
f\left(A, \delta^{\prime}\right) & =\sup \left\{b>0 ; \exists \tau \in I M ; P(A(\tau)) \geqslant b, \int \Phi\left(\eta_{1} e_{\tau}\right) d P \leqslant \delta^{\prime} b\right\} \\
& =\sup \left\{P(A(\tau)) ; \tau \in I M ; \int \Phi\left(\eta_{1} e_{\tau}\right) d P \leqslant \delta^{\prime} P(A(\tau))\right\}
\end{aligned}
$$

This is an increasing function of $\boldsymbol{\delta}^{\prime}$. 
Given $\varepsilon^{\prime}, \gamma^{\prime}>0, s^{\prime} \in J$, let $F\left(\varepsilon^{\prime}, \gamma^{\prime}, s^{\prime}\right)$ denote the collection of the finite adapted families $A=\left(A_{t}\right)$ such that the following holds:

$$
A_{t} \neq \varnothing \Rightarrow t \geqslant s^{\prime}, \quad P\left(\bigcup A_{t}\right)>\varepsilon^{\prime}, \quad \sum P\left(A_{t}\right) \leqslant\left(1+\gamma^{\prime}\right) P\left(\bigcup A_{t}\right) .
$$

Write

$$
g\left(\varepsilon^{\prime}, \gamma^{\prime}, s^{\prime}, \delta^{\prime}\right)=\operatorname{Inf}\left\{f\left(A, \delta^{\prime}\right) ; A \in F\left(\varepsilon^{\prime}, \gamma^{\prime}, s^{\prime}\right)\right\} .
$$

This function is increasing in $\varepsilon^{\prime}, \delta^{\prime}$ and $s^{\prime}$, decreasing in $\gamma^{\prime}$. Let

$$
h\left(\varepsilon^{\prime}, \delta^{\prime}\right)=\sup _{s^{\prime} \in i, \gamma^{\prime}>0} g\left(\varepsilon^{\prime}, \gamma^{\prime}, s^{\prime}, \delta^{\prime}\right) .
$$

If $h(\delta, \delta / 2)>0$, the result is true. Actually, in this case it is enough to take $b_{2}=h(\delta, \delta / 2) / 2$, and $s_{2}, \gamma_{2}$, with $g\left(\delta, \gamma_{2}, s_{2}, \delta / 2\right)>b_{2}$.

We now suppose $h(\delta, \delta / 2)=0$. Let

$$
\varepsilon_{0}=\inf \left\{\varepsilon^{\prime} \geqslant 0 ; \forall \delta^{\prime}>0, h\left(\varepsilon^{\prime}, \delta^{\prime}\right)>0\right\} .
$$

Lemma 31 means that, for each $\delta^{\prime}>0$, we have $h\left(\varepsilon, \delta^{\prime}\right)>0$, so we have $\delta \leqslant \varepsilon_{0}<\varepsilon$. Since $\varepsilon_{0}-\delta / 2<\varepsilon_{0}$, there is $\delta^{\prime}>0$ with $h\left(\delta_{0}-\delta / 2, \delta^{\prime}\right)=0$. Let $b_{2}=$ $h\left(\varepsilon_{0}+\delta / 2, \delta^{\prime} / 4\right) / 4>0$. Let $s_{3} \in j, \gamma_{2}>0$ such that

$$
2 b_{2}<g\left(\varepsilon_{0}+\delta / 2,2 \gamma_{2}, s_{3}, \delta^{\prime} / 4\right) \leqslant 4 b_{2} \text {. }
$$

Since $h\left(\varepsilon_{0}-\delta / 2, \delta^{\prime}\right)=0$, we have $g\left(\varepsilon_{0}-\delta / 2, \gamma_{2}, s_{3}, \delta^{\prime}\right)=0$. So there is $B=\left(B_{t}\right)$ $\in F\left(\varepsilon_{0}-\delta / 2, \gamma_{2}, s_{3}\right)$ such that $f\left(B, \delta^{\prime}\right)<b_{2}$. Let $I=\left\{t ; B_{t} \neq \varnothing\right\}$. Let $U=\bigcup B_{t}$. We have $P(U) \geqslant \varepsilon_{0}-\delta / 2$. We have $P(U) \leqslant \varepsilon_{0}+\delta / 2$, for otherwise $B \in$ $F\left(\varepsilon_{0}+\delta / 2, \gamma_{2}, s_{3}\right)$, which is impossible because

$$
f\left(B, \delta^{\prime}\right)<b_{2}<g\left(\varepsilon_{0}+\delta / 2,2 \gamma_{2}, s_{3}, \delta^{\prime} / 4\right)<g\left(\varepsilon_{0}+\delta / 2, \gamma_{2}, s_{3}, \delta^{\prime}\right) .
$$

Let $T=\Omega \backslash U$, so $P(T) \geqslant 1-\varepsilon-\delta$. Let $s_{2} \in J$ with $s_{2} \geqslant I$.

Let $A=\left(A_{t}\right)$ be an adapted family such that (20) and (21) hold. Let $A_{t}^{\prime}=B_{t}$ for $t \in I, A_{t}^{\prime}=A_{t} \backslash U=A_{t} \cap T$ for $t \geqslant s_{2}$, and $A_{t}^{\prime}=\varnothing$ otherwise. It follows from (20) that $P\left(\bigcup_{t \geqslant s_{3}} A_{t}^{\prime}\right) \geqslant \varepsilon_{0}+\delta / 2$. Also,

$$
\begin{aligned}
\sum_{t \geqslant s_{3}} P\left(A_{t}^{\prime}\right)= & \sum_{t \in I} P\left(B_{t}\right)+\sum_{t \geqslant s_{2}} P\left(A_{t}^{\prime}\right) \\
\leqslant & \left(1+\gamma_{2}\right)\left[P(U)+P\left(\bigcup_{t \geqslant s_{2}} A_{t}\right)\right]-\sum_{t \geqslant s_{2}} P\left(A_{t} \cap U\right) \\
\leqslant & \left(1+\gamma_{2}\right)\left[P(U)+P\left(\bigcup_{t \geqslant s_{2}} A_{t} \backslash U\right)\right] \\
& +\left(1+\gamma_{2}\right) P\left(\bigcup_{t \geqslant s_{2}} A_{t} \cap U\right)-\sum_{t \geqslant s_{2}} P\left(A_{t} \cap U\right) \\
& \left(1+2 \gamma_{2}\right) P\left(\bigcup A_{t}^{\prime}\right) .
\end{aligned}
$$

It follows that

$$
A^{\prime}=\left(A_{t}^{\prime}\right) \in F\left(\varepsilon_{0}+\delta / 2,2 \gamma_{2}, s_{3}\right) \text {. }
$$

The definition of $g\left(\varepsilon_{0}+\delta / 2,2 \gamma_{2}, s_{3}, \delta^{\prime} / 4\right)$ shows that there is a $c>0$ with

$$
2 b_{2} \leqslant c \leqslant g\left(\varepsilon_{0}+\delta / 2,2 \gamma_{2}, s_{3}, \delta^{\prime} / 4\right) \leqslant 4 b_{2}
$$


and $\tau^{\prime} \in I M$ with

$$
P\left(A^{\prime}\left(\tau^{\prime}\right)\right)=c \geqslant 2 b_{2}, \quad \int \Phi\left(\eta_{1} e_{\tau^{\prime}}\right) d P \leqslant c \delta^{\prime} / 4 \leqslant \delta b_{2} .
$$

Let $\tau_{1} \in I M$ be given by $\left\{\tau_{1}=t\right\}=\left\{\tau^{\prime}=t\right\}$ for $t \in I$ and $\left\{\tau_{1}=t\right\}=\varnothing$ otherwise. We have $\int \Phi\left(\eta_{1} e_{\tau_{1}}\right) d P \leqslant \delta b_{2}$. Since $f\left(B, \delta^{\prime}\right)<b_{2}$, this means that $P\left(B\left(\tau_{1}\right)\right) \leqslant$ $b_{2}$. Let $\tau \in I M$ be given by $\{\tau=t\}=\left\{\tau^{\prime}=t\right\}$ for $t \geqslant s_{2}$ and $\{\tau=t\}=\varnothing$ otherwise. Then $A^{\prime}\left(\tau^{\prime}\right) \subset B\left(\tau_{1}\right) \cup A(\tau)$, so we have $P(A(\tau)) \geqslant b_{2}$. Since $\int \Phi\left(\eta_{1} e_{\tau}\right) d P \leqslant \delta b_{2}$, the proof is complete.

We can start the proof of Theorem 8.

Proposition 33. Assume that $J$ contains a countable cofinal set and that $L^{\Psi}$-bounded martingales converge essentially. Then for each $\varepsilon>0$ there are $\eta=\eta(\varepsilon)$ and a set $\Omega_{\varepsilon}$ with $P\left(\Omega_{\varepsilon}\right) \geqslant 1-\varepsilon$ such that, for each adapted family $A=\left(A_{t}\right)$ and each $\delta>0$, there is $\tau \in I M$ with $P\left(\left(A^{*} \cap \Omega_{\varepsilon}\right) \backslash A(\tau)\right) \leqslant \delta$ and $\int \Phi\left(\eta e_{\tau}\right) d P \leqslant \delta$.

Proof. First step. Let $\eta=\eta_{1}(\varepsilon / 2)$. According to Lemma 32, for each $\eta$ there are $s(n) \in J, \gamma(n), b(n)>0$ and a set $T_{n} \in \Sigma$ with $P\left(T_{n}\right) \geqslant 1-\varepsilon / 2-2^{-n}$ such that for every adapted family of sets $\left(A_{t}\right)$ satisfying

$$
\begin{gathered}
P\left(\bigcup_{t \geqslant s(n)} A_{t} \cap T_{n}\right) \geqslant 2^{-n}, \\
\sum_{t \geqslant s(n)} P\left(A_{t}\right) \leqslant(1+\gamma(n)) P\left(\bigcup_{t \geqslant s(n)} A_{t}\right),
\end{gathered}
$$

there is $\tau \in I M$ with $P(A(\tau)) \geqslant b(n)$ and $\int \Phi\left(\eta e_{\tau}\right) d P \leqslant 2^{-n} b(n)$.

Let $f$ be a cluster point of the sequence $1_{T_{n}}$ for the weak topology of $L^{2}(P)$. We have $f \leqslant 1$ and $\int f d P \geqslant 1-\varepsilon / 2$. Let $\Omega_{\varepsilon}=\left\{f>\frac{1}{2}\right\}$, so $P\left(\Omega_{\varepsilon}\right) \geqslant 1-\varepsilon$.

Second step. Since $L^{\infty} \subset L^{\Psi}$, all $L^{\infty}$ bounded martingales converge essentially. So condition $V_{1}$ of Krickeberg holds [5], that is for each adapted family $A$, and for each $\gamma>0$, there is $\tau \in I M$ with $P(A(\tau)) \geqslant P(A)-\gamma$ and $\left\|e_{\tau}\right\|_{1} \leqslant \gamma$.

Third step. Let $A$ be an adapted family with $P\left(A^{*} \cap \Omega_{\varepsilon}\right)>0$, and let $\delta>0$. Since $\int_{A^{*} \cap \Omega_{\varepsilon}} f d P>P\left(A^{*} \cap \Omega_{\varepsilon}\right) / 2$, there is $n$ with $2^{-n}<\delta, 2^{-n+2}<P\left(A^{*} \cap \Omega_{\varepsilon}\right)$ such that $P\left(A^{*} \cap \Omega_{\varepsilon} \cap T_{n}\right) \geqslant P\left(A^{*} \cap \Omega_{\varepsilon}\right) / 2$.

By induction over $k$, we construct $\tau_{k} \in I M$ such that

$$
P\left(A\left(\tau_{k}\right)\right) \geqslant k b(n), \quad \int \Phi\left(\eta e_{\tau_{k}}\right) d P \leqslant k \delta b(n) .
$$

The induction continues as long as $P\left(\left(A^{*} \cap T_{n}\right) \backslash A\left(\tau_{k}\right)\right) \geqslant 2^{-n}$, so it stops in a finite number of steps, and produces $\tau \in I M$ with $P(A(\tau)) \geqslant P\left(A^{*} \cap \Omega_{\varepsilon}\right) / 4$ and $\int \Phi\left(\eta e_{\tau}\right) d P \leqslant \delta P(A(\tau)) \leqslant \delta$.

The second step shows that there is $\tau^{\prime} \in I M$ with $P\left(A\left(\tau^{\prime}\right) \cap T_{n}\right)>b(n)$ and $\left\|e_{\tau^{\prime}}\right\|_{1} \leqslant \gamma(n)$. In other words, the family $\left(A_{t}^{\prime}\right)$ given by $A_{t}^{\prime}=A_{t} \cap\left\{\tau^{\prime}=t\right\}$ satisfies (22) and (23). The existence of $\tau_{1}$ follows from the first step.

Assume now that $\tau_{k}$ has been constructed, and that $P\left(\left(A^{*} \cap T_{n}\right) \backslash A\left(\tau_{k}\right)\right)>2^{-n}$. Let $s \geqslant \tau_{k}$. Define $B_{t}=A_{t} \backslash A\left(\tau_{k}\right)$ for $t \geqslant s, B_{t}=\varnothing$ otherwise. Then $P\left(B^{*} \cap T_{n}\right)>$ $2^{-n}$, so the second step shows that there is $\tau^{\prime} \in I M$ with $P\left(B\left(\tau^{\prime}\right) \cap T_{n}\right)>2^{-n}$ and 
$\left\|e_{\tau^{\prime}}\right\|_{1} \leqslant \gamma(n)$. In particular, the family $\left(A_{t}^{\prime}\right)$ given by $A_{t}^{\prime}=A_{t} \cap\left\{\tau^{\prime}=t\right\}$ satisfies (22) and (23), so there is $\tau^{\prime \prime}$ with $P\left(A^{\prime}\left(\tau^{\prime \prime}\right)\right)>b(n)$ and $\int \Phi\left(\eta e_{\tau^{\prime \prime}}\right) d P \leqslant \delta b(n)$. We now define $\left\{\tau_{k+1}=t\right\}=\left\{\tau_{k}=t\right\}$ for $t \leqslant s,\left\{\tau_{k+1}=t\right\}=\left\{\tau^{\prime \prime}=t\right\}$ for $t \geqslant s$, $\left\{\tau_{k+1}=t\right\}=\varnothing$ otherwise, and the induction continues.

Fourth step. Using one more standard exhaustion, that we leave to the reader, we find that there is $\tau \in I M$ with $P(A(\tau)) \geqslant P\left(A^{*} \cap \Omega_{\varepsilon}\right)-\delta$ and $\int \Phi\left(\eta e_{\tau}\right) d P \leqslant \delta$. This is formally weaker than the property we look for. However, there is $s \in J$ and $C \subset \Sigma_{s}$ with $P\left(C \cap A^{*} \cap \Omega_{\varepsilon}\right) \geqslant P\left(A^{*} \cap \Omega_{\varepsilon}\right)-\delta$ and $P\left(C \backslash \Omega_{\varepsilon}\right) \leqslant \varepsilon$. Define $B_{t}=$ $A_{t} \cap C$ for $t \geqslant s, B_{t}=\varnothing$ otherwise. The above result applied to $B$ yields a $\tau \in I M$ with $\int \Phi\left(\eta e_{\tau}\right) d P \leqslant \delta$ and $P\left(\left(A^{*} \cap \Omega_{\varepsilon}\right) \backslash A(\tau)\right) \leqslant 3 \delta$. The proof is complete.

We now prove Theorem 8 . The preceding proposition asserts that condition $D_{\Phi}$ is necessary when $J$ contains a countable cofinal set. Since we assume $L^{\Psi} \neq L^{1}$, we consider only martingales of the type $\left(E^{t}(f)\right)$ for $f \in L^{\Psi}$; the fact that condition $D_{\Phi}$ is sufficient will follow from the proof of Proposition 24 and the following

Proposition 34. Assume that condition $D_{\Phi}$ holds. Let $f \in L^{\Psi}, f \geqslant 0$. Let $X_{t}=E^{t}(f)$. Then for $\lambda>0$ we have $P\left(X^{*}>\lambda\right) \leqslant E(f) / \lambda$.

Proof. Let $\alpha>0$. There is $\gamma>0$ such that $\int \Psi\left(f \alpha^{-1} \gamma\right) d P<\infty$. Since we assume (5), we have $\Phi(x)>0$ for $x>0$, so for each $u, \gamma \rightarrow \gamma^{-1} \Psi(u \gamma)$ is increasing from zero. Lebesgue's theorem shows that there is $\gamma>0$ such that $\int \gamma^{-1} \Psi\left(f \alpha^{-1} \gamma\right) d P \leqslant 1$. Let $\lambda>0$, and let $0<\beta<\lambda$. Let $A_{t}=\left\{X_{t}>\beta\right\}$. Then $P\left(X^{*}>\lambda\right) \leqslant P\left(A^{*}\right)$. Let $0<\varepsilon<P\left(A^{*}\right)$. Then condition $D_{\Phi}$ gives $\eta(\varepsilon)$ and $\tau \in I M$ such that $\int \gamma^{-1} \Phi\left(\eta(\varepsilon) e_{\tau}\right) d P \leqslant 1$ and $P\left(A^{*} \backslash A(\tau)\right)<\varepsilon$. We can assume $\{\tau=t\} \subset$ $A_{t}$ for each $t$. We get

$$
E\left(S_{\tau} f\right)=\sum E\left(f 1_{\{\tau=i\}}\right)=\sum E\left(X_{i} 1_{\{\tau=i\}}\right) \geqslant \beta P(A(\tau)) .
$$

On the other hand, $\gamma^{-1} \Phi(x)$ and $\gamma^{-1} \Psi(x \gamma)$ are conjugate Young functions, so Young's inequality (1) gives

$$
E\left(e_{\tau} \eta(\varepsilon) f \alpha^{-1}\right) \leqslant 1, \quad \text { so } E\left(e_{\tau} f\right) \leqslant \alpha / \eta(\varepsilon)
$$

so we get

$$
E\left(S_{\tau} f\right) \leqslant E(f)+E\left(e_{\tau} f\right) \leqslant E(f)+\alpha / \eta(\varepsilon)
$$

and

$$
P\left(\left\{X^{*}>\lambda\right\}\right) \leqslant P\left(A^{*}\right) \leqslant \varepsilon+\beta^{-1}(E(f)+\alpha / \eta(\varepsilon)) .
$$

Letting $\beta, \varepsilon$ be fixed, we let $\alpha$ go to zero. We then let $\varepsilon$ go to zero and $\beta$ go to $\lambda$ to get the required inequality. The proof is complete.

5. Proof of Theorem 9. Since $\int \Phi\left(e_{\tau} / n\right) d P \leqslant 1$ implies $\left\|e_{\tau}\right\|_{\Phi} \leqslant 2 n$, condition $D_{\Phi}$ implies condition $C_{\Phi}$. So we have already proved that when $L^{\Psi} \neq L^{1}$, condition $C_{\Phi}$ is necessary when $J$ contains a countable cofinal set. Actually using our techniques, the direct proof that $C_{\Phi}$ is necessary is much easier than the proof that $D_{\Phi}$ is necessary. (But, as Theorem 11 shows, $C_{\Phi}$ is not sufficient in general). We suppose now $L^{\Psi}=L^{1}$, and we prove in that case that $D_{\Phi}$ is necessary when $J$ contains a countable cofinal set. 
LEMMA 35. Let $I \subset J$ be a finite set, and for each $i \in I$, let $A_{i}$ be a $\Sigma_{i}$-measurable set. Let $a>0$. Assume that for each family $\left(\xi_{i}\right)_{i \in I}$ of functions, $\xi_{i} \geqslant 0, \xi_{i}=0$ outside $A_{i}, \xi_{i}$ is bounded $\Sigma_{i}$-measurable, and $\left\|\sum_{i \in I} \xi_{i}\right\|_{1}=1$, we have $\left\|\Sigma_{i \in I} \xi_{i}\right\|_{\infty} \geqslant a$. Then for each $\gamma>0$ there exists $f \in L^{1}$ with $\|f\|_{2} \leqslant 1 / a, f \geqslant 0$, such that, for $i \in I$, we have $P\left(A_{i} \backslash A_{i}^{\prime}\right) \leqslant \gamma$, where $A_{i}^{\prime}=\left\{E^{i}(f) \geqslant \frac{1}{2}\right\}$.

Proof. We denote by $C_{1}$ the set of functions $\sum_{i \in I} \xi_{i}$ where $\xi_{i}$ is bounded, $\Sigma_{i}$-measurable, $\xi_{i} \geqslant 0, \xi_{i}=0$ outside $A_{i},\left\|\sum \xi_{i}\right\|_{1}=1,\left\|\sum \xi_{i}\right\|_{\infty} \leqslant 1 / \gamma$.

We denote by $C_{2}$ the set of $\xi$ with sup $\xi \leqslant a / 2$. Then $C_{1}, C_{2}$ are convex. Moreover, $C_{1}$ is $\sigma\left(L^{\infty}, L^{1}\right)$-compact and $C_{2}$ is $\sigma\left(L^{\infty}, L^{1}\right)$-closed. The theorem of Hahn-Banach gives $f \in L^{1}$ with $f<\frac{1}{2}$ on $C_{2}$ and $f>\frac{1}{2}$ on $C_{1}$. Since $f<\frac{1}{2}$ on $C_{2}$, we have $f \geqslant 0$ and $\|f\|_{1} \leqslant 1 / a$. Suppose, if possible, that for some $i$ we have $P\left(B_{i}\right)>\gamma$, where $B_{i}=A_{i} \backslash A_{i}^{\prime}$. Then the function $h=P\left(B_{i}\right)^{-1} 1_{B_{i}}$ belongs to $C_{1}$, so $E(f h)>\frac{1}{2}$. However, $E(f h)=P\left(B_{i}\right)^{-1} \int_{B_{i}} E^{i}(f) \leqslant \frac{1}{2}$. This contradiction proves the lemma.

LEMMA 36. Assume that $J$ contains a countable cofinite set and that for each $f \in L^{1}$ the martingale $\left(E^{t}(f)\right)$ converges essentially. Then for each $\varepsilon>0$ there is $s \in J$ and $N_{\varepsilon}>0$ such that, for each finite set $I \subset J$ with $s \leqslant I$, and each family $\left(A_{i}\right)_{i \in I}, A_{i}$ is $\Sigma_{i}$-measurable, $P\left(\cup A_{i}\right) \geqslant \varepsilon$, there exist bounded functions $\xi_{i}$, $\xi_{i}$ is $\Sigma_{i}$-measurable, $\xi_{i} \geqslant 0, \xi_{i}=0$ outside $A_{i},\left\|\sum \xi_{i}\right\|_{1}=1,\left\|\sum \xi_{i}\right\|_{\infty} \leqslant N_{\varepsilon}$.

Proof. Otherwise there is $\varepsilon>0$ such that, for each $s \in J$ and $N>0$, there is a finite set $I \subset J$, with $s \leqslant I$ and a family $\left(A_{i}\right)_{i \in I}$ of $\Sigma_{i}$-measurable sets, with $P\left(\cup A_{i}\right) \geqslant \varepsilon$, such that for each family $\xi_{i}$ of bounded functions $\xi_{i}, \xi_{i} \geqslant 0, \xi_{i}=0$ outside $A_{i},\left\|\sum \xi_{i}\right\|_{1}=1$, then $\left\|\sum \xi_{i}\right\|_{\infty} \geqslant N$.

Let $\left(s_{k}\right)$ be a cofinal sequence in $I$. Using the preceding statement and Lemma 35 , we construct for each $k$ a finite set $I_{k}$ with $s_{k} \leqslant I_{k}$ and $f_{k} \in L^{1}, f \geqslant 0,\left\|f_{k}\right\|_{1} \leqslant \varepsilon 2^{-k-2}$ such that

$$
P\left(\bigcup_{i \in I_{k}}\left\{E^{i}\left(f_{k}\right)>\frac{1}{2}\right\}\right) \geqslant \varepsilon-\frac{1}{k} .
$$

Let $f=\sum f_{k}$. Then $\|f\|_{1} \leqslant \varepsilon / 4$. Let $X_{t}=E^{t}(f)$. From (25) we see that $P\left(X^{*} \geqslant \frac{1}{2}\right)$ $\geqslant \varepsilon$. On the other hand, $P\left(X_{*} \geqslant \frac{1}{2}\right) \leqslant \varepsilon / 2$, so $X^{*} \neq X_{*}$. This contradiction concludes the proof.

LEMMA 37. Assume that $J$ contains a countable cofinite set and that for each $f \in L^{1}$ the martingale $\left(E^{t}(f)\right)$ converges essentially. Then for each $\varepsilon>0$ there is $M_{\varepsilon}>0$, $b>0, s \in J$ and $\gamma>0$ such that, for each finite adapted family $\left(A_{t}\right)$,

$$
P\left(\bigcup_{t>s} A_{t}\right)>\varepsilon, \quad \sum_{t>s} P\left(A_{t}\right) \leqslant(1+\gamma) P\left(\bigcup_{t>s} A_{t}\right),
$$

there exists $\tau \in I M$ with $P(A(\tau))>b$ and $\left\|S_{\tau}\right\|_{\infty} \leqslant M_{\varepsilon}$.

Proof. Let $N_{\varepsilon}$ and $s$ be as in Lemma 36. Let $b=1 / 4 N_{\varepsilon}, \gamma=1 / 2 N_{\varepsilon}$. Let $\left(A_{t}\right)$ be an adapted family satisfying (26). We can assume there is $I \geqslant s$ such that $A_{t}=\varnothing$ for $t \notin I$. Let

$$
C=\left\{\omega ; \exists i, j \in I, i \neq j, \omega \in A_{i} \cap A_{j}\right\}
$$


Then $P(C) \leqslant 1 / 2 N_{\varepsilon}$. Let $\left(\xi_{i}\right)_{i \in J}$ be the functions given by Lemma 36 , and let $\xi=\sum \xi_{i}$. Since $\|\xi\|_{\infty} \leqslant N_{\varepsilon}$, we have $\int_{C} \xi d P \leqslant \frac{1}{2}$, so $\int_{\Omega \backslash C} \xi d P \geqslant \frac{1}{2}$. It follows that if $H=\left\{\xi>\frac{1}{4}\right\} \backslash C$ we get $P(H) \geqslant 1 / 4 N_{\varepsilon}$. For $\omega \in H$, there is a unique $i \in I$ with $\xi_{i}(\omega)>0$, so $\xi_{i}(\omega) \geqslant \frac{1}{4}$. Define $\tau \in I M$ by $\{\tau=i\}=\left\{\xi_{i} \geqslant \frac{1}{4}\right\}$ for $i \in I,\{\tau=i\}$ $=\varnothing$ otherwise. We have shown that $P(A(\tau)) \geqslant P(H) \geqslant b$. On the other hand, $S_{\tau} \leqslant 4 \xi$, so $\left\|S_{\tau}\right\|_{\infty} \leqslant 4 N_{\varepsilon}$. The proof is complete.

At this point, the fact that condition $V_{1}$ holds and the standard exhaustion procedure make it clear that condition $C_{\Phi}$ is necessary for the convergence of equi-integrable $L^{1}$-bounded martingales. The proof that condition $D_{\Phi}$ is also necessary follows the line of Lemmas 32 and 33 with some simplifications.

It remains to show that condition $C_{\Phi}$ implies the convergence of $L^{\Psi}$-bounded martingales if $\Psi$ satisfies the $\Delta_{2}$ condition.

Proposition 38. Assume that condition $C_{\Phi}$ holds. Let $s \in J, B \in \Sigma_{s}$. Then for each positive submartingale $\left(X_{t}\right)$ and $\lambda>0$ we have

$$
P\left(\left\{X^{*} \geqslant \lambda\right\} \cap B\right) \leqslant \varepsilon+\left(2 M_{\varepsilon} / \lambda\right) \sup _{t}\left\|X_{t} 1_{B}\right\|_{\Psi} \text {. }
$$

Proof. We can assume $P\left(\left\{X^{*} \geqslant \lambda\right\} \cap B\right)>\varepsilon$. Let $A_{t}=\left\{X_{t} \geqslant \lambda / 2\right\} \cap B$ for $t \geqslant s$. Then $\varepsilon<P\left(\left\{X^{*} \geqslant \lambda\right\} \cap B\right) \leqslant P\left(A^{*}\right)$. From condition $C_{\Phi}$, there is a $\tau \in I M$ such that $P\left(A^{*} \backslash A(\tau)\right) \leqslant \varepsilon$ and $\left\|S_{\tau}\right\|_{\Phi} \leqslant M_{\varepsilon}$. Let $t \geqslant \tau$. We have

$$
E\left(X_{t} S_{\tau} 1_{B}\right) \geqslant \sum E\left(X_{t} 1_{\{\tau=i\}} 1_{B}\right) \geqslant \sum E\left(X_{t} 1_{\{\tau=i\} \cap B}\right) \geqslant \lambda P(A(\tau)) .
$$

On the other hand, $E\left(X_{t} S_{\tau} 1_{B}\right) \leqslant\left\|X_{t} 1_{B}\right\|_{\Psi} M_{\varepsilon}$, so we get

$$
P\left(A^{*}\right) \leqslant \varepsilon+\left(2 M_{\varepsilon} / \lambda\right) \sup _{t}\left\|X_{t} 1_{B}\right\|_{\Psi} \text {. }
$$

Proposition 39. Assume that $\Psi$ satisfies the $\Delta_{2}$ condition. Assume that for every $s \in J$, every $B \in \Sigma_{s}$, every positive submartingale $\left(X_{t}\right)$, we have for $\lambda>0$

$$
P\left(\left\{X^{*}>\lambda\right\} \cap B\right) \leqslant \varepsilon+\left(2 M_{\varepsilon} / \lambda\right) \sup _{t}\left\|X_{t} 1_{B}\right\|_{\Psi} .
$$

Then every $L^{\Psi}$-bounded martingale converges.

Proof. Let $f \in L^{\Psi}$ and $\varepsilon>0$. Denote by $f_{a}$ the truncation of $f$ at $-a$ and $a$. Since $\Psi$ satisfies the $\Delta_{2}$ condition, $\left\|f-f_{a}\right\|_{\Psi} \rightarrow 0$ when $a \rightarrow \infty$. It follows that there are $s \in J$ and $g \in L^{\Psi}, g$ is $\Sigma_{s}$-measurable, with $\|f-g\|_{\Psi}<\varepsilon / 2 M_{\varepsilon}$. Let $Y_{t}=E^{t}(|f-g|)$. From (27) we get $P\left(Y^{*}>\varepsilon\right) \leqslant 2 \varepsilon$. Since $\left|E^{t}(f)-E^{t}(g)\right| \leqslant Y_{t}$ and $E^{t}(g)=g$ for $t \geqslant s$, we see that the martingale $\left(E^{t}(f)\right)$ converges essentially. If $L^{\Psi} \neq L^{1}$, all the $L^{\Psi}$-bounded martingales are equi-integrable, so are of this type. We now investigate the case $L^{\Psi}=L^{1}$. Let $\Xi$ denote the union of all the algebras $\Sigma_{t}$. For $A \in \Xi$, let $m(A)=\lim _{t} \int_{A} X_{t} d P$. This is a finitely additive measure of bounded variation. As in [11], we write $m=m_{1}+m_{2}$ where $m_{1}$ is absolutely continuous and $m_{2}$ is singular. Let $m_{1}(A)=\int_{A} f d P$. We have shown the convergence of $\left(E^{t}(f)\right)$. It remains to show that $Y_{t}=X_{t}-E^{t}(f)$ converges essentially to zero. Let $Z_{t}=\left|Y_{t}\right|$. This is a submartingale. Let $\varepsilon>0$. Let $s \in J$ and $B \in J$ with $P(B) \geqslant 1-\varepsilon$ and $\left|m_{2}\right|(B)<$ $\varepsilon / 2 M_{\varepsilon}$. Since $E\left(Z_{t} 1_{B}\right) \leqslant\left|m_{2}\right|(B)$, from (27) we get $P\left(\left\{Z^{*} \geqslant \varepsilon\right\} \cap B\right) \leqslant 2 \varepsilon$, so $P\left(Z^{*} \geqslant \varepsilon\right) \leqslant 3 \varepsilon$, and this concludes the proof. 


\section{Proof of Theorem 10.}

LEMMA 40. If $\Phi$ does not satisfy condition Exp, for each $a>0$ there is an integer $l$ with $\Phi(2 l) \geqslant a^{l} \Phi(l)$.

Proof. If for each $n$ we have $\Phi\left(2^{n+1}\right) \leqslant a^{2^{n}} \Phi\left(2^{n}\right)$, we get $\Phi\left(2^{n}\right) \leqslant a^{2^{n}} \Phi(1)$ for each $n$, so for each $u \geqslant 1$ we have $\Phi(u) \leqslant a^{2 u} \Phi(1)$, which implies condition Exp.

We now start the construction. Since the details differ whether $\Phi$ is always finite or not, we assume $\Phi$ always finite, leaving the other (simpler) case to the reader. By the lemma, there is a sequence $\left(l_{n}\right)$ of integers with

$$
\Phi\left(2 l_{n}\right) \geqslant 2^{n+3} 2^{2(n+4) l_{n}} \Phi\left(l_{n}\right) .
$$

We can assume $l_{n} \rightarrow \infty$ and $\Phi\left(l_{n}\right) \geqslant 2^{3}$. Let $k_{n}=2^{n+4} l_{n}$. Let $G_{n}=\left\{1, \ldots, k_{n}\right\}$, $H_{n}=\left\{1, \ldots, l_{n}\right\}$. Let $U_{n}$ be the uniform probability on $G_{n}$. Let $M_{n}=G_{n}^{H_{n}}$, and $Q_{n}$ be the power of $U_{n}$ on $M_{n}$. Let $L_{n}=G_{n} \cup M_{n}$. Let $b_{n}=2^{-n} / \Phi\left(l_{n}\right)$, so $b_{n} \leqslant 2^{-n-5}$. On $L_{n}$, consider the probability $P_{n}$ given by

$$
P_{n}(B)=\left(1-b_{n}\right) U_{n}\left(B \cap G_{n}\right)+b_{n} Q_{n}\left(B \cap M_{n}\right) .
$$

For $i \leqslant k_{n}$, let

$$
C_{n, i}=\{i\} \cup\left\{y \in M_{n}, \exists p \in H_{n} ; y(p)=i\right\} \text {. }
$$

Let $L=\prod L_{n}$, and $P$ be the product probability. Then $(L, \mathscr{B}, P)$ is isomorphic to $([0,1], \mathscr{B}, \lambda)$ where $\mathscr{B}$ is the Borel $\sigma$-algebra. We denote by $\Sigma_{n}$ the $\sigma$-algebra of sets that depend only on the first $n-1$ coordinates. Let $B_{n, i}=\left\{z=\left(z_{n}\right) \in L ; z_{n} \in\right.$ $\left.C_{n, i}\right\}$. We denote by $\Sigma_{n, i}$ the $\sigma$-algebra generated by $\Sigma_{n}$ and $B_{n, i}$. Let $J=\{(n, i) ; i \in$ $\left.H_{n}\right\}$. For $\left(n^{\prime}, i^{\prime}\right) \in J$, we say that $(n, i)<\left(n^{\prime}, i^{\prime}\right)$ if either $n<n^{\prime}$ or $(n, i)=\left(n^{\prime}, i^{\prime}\right)$. The map $t \rightarrow \Sigma_{t}$ is increasing.

We first show that condition $D_{\Phi}$ holds. Let $\left(A_{t}\right)$ be an adapted family of sets. Let $\varepsilon>0$. Let $n_{0}$ with $2^{-n_{0}} \leqslant \varepsilon$. Let $A_{t}^{\prime \prime}=\varnothing$ if $t=(m, i)$ for $m \leqslant n_{0}$ and $A_{t}^{\prime \prime}=A_{t}$ otherwise. Let $A_{t}^{\prime}=A_{t}^{\prime \prime} \backslash \bigcup_{s<t} A_{s}^{\prime}$. For each, let $C_{n}=\bigcup_{i \in H_{n}} A_{n, i}^{\prime}$. Then the sets $C_{n}$ are disjoint, and $\cup C_{n} \supset A^{*}$. Let $q$ be such that $P\left(A^{*} \backslash \cup_{n \leqslant q} C_{n}\right) \leqslant \varepsilon$. Define $\tau \in I M$ by $\{\tau=(n, i)\}=A_{n, i}^{\prime}$ for $n \leqslant q$, and $\{\tau=(n, i)\}=\varnothing$ otherwise. Then $A(\tau)=$ $\bigcup_{n \leqslant q} C_{n}$, so $P\left(A^{*} \backslash A(\tau)\right) \leqslant \varepsilon$. Let $f=\sum_{i \in H_{n}} 1_{B_{n, i}}, g_{n}=f-f \wedge 1$. Then for $x=\left(x_{n}\right)$ $\in L, g_{n}(x)=0$ except when $x_{n} \in M_{n}$, in which case $g_{n} \leqslant l_{n}$, so $\int \Phi\left(g_{n}\right) d P \leqslant$ $b_{n} \Phi\left(l_{n}\right) \leqslant 2^{-n}$. But one sees that $e_{\tau} \leqslant g_{n}$ on $C_{n}$. Since $C_{n}=\varnothing$ for $n \leqslant n_{0}$, we get

$$
\int \Phi\left(e_{\tau}\right) d P \leqslant \sum_{n>n_{0}} \int_{C_{n}} \Phi\left(g_{n}\right) d P \leqslant 2^{-n_{0}} \leqslant \varepsilon
$$

so condition $D_{\Phi}$ holds.

We now show that condition $V_{\Phi}$ fails. Let $A_{n, i}=B_{n, i}$ for each $n, i$. Then $A^{*}=L$. Let $\tau \in I M$ be such that $P(A(\tau))>\frac{1}{2}$. We are going to show that $\left\|e_{\tau}\right\|_{\Phi} \geqslant \frac{1}{2}$.

For each $n$, let

$$
B_{n}=\bigcup_{i \in H_{n}} B_{n, i} \cap\{\tau=(n, i)\}
$$


We have $A(\tau)=\cup_{n} B_{n}$, so there exists $n$ with $P\left(B_{n}\right) \geqslant 2^{-n-1}$. Let $\mathscr{Y}$ be the family of atoms $Y$ of $\Sigma_{n}$ such that $P\left(Y \cap B_{n}\right) \geqslant 2^{-n-2} P(Y)$. Then the union of $\mathscr{Y}$ has probability $\geqslant 2^{-n-2}$. Let us fix $Y \in \mathscr{Y}$. Let $l=$ card $K$, where

$$
K=\left\{i \in H_{n} ; Y \cap B_{n, i} \subset\{\tau=(n, i)\}\right\} .
$$

We have

$$
P\left(Y \cap B_{n}\right) \leqslant P(Y)\left(b_{n}+l\left(1-b_{n}\right) / k_{n}\right) .
$$

Since $b_{n} \leqslant 2^{-n-3}$, it follows that $l \geqslant 2^{-n-3} k_{n} \geqslant 2 l_{n}$. Let

$$
N=\left\{y \in M_{n} ; \text { the numbers } y(p) \text { for } p \in H_{n} \text { are distinct and in } K\right\} .
$$

Since $l \geqslant 2 l_{n}$, we have

$$
P_{n}(N) \geqslant b_{n}\left(\frac{l}{k_{n}} \frac{l-1}{k_{n}} \cdots \frac{l-l_{n}+1}{k_{n}}\right) \geqslant b_{n}\left(l / 2 k_{n}\right)^{l_{n}} \geqslant b_{n}\left(2^{-n-4}\right)^{l_{n}} .
$$

Let $N^{\prime}=\left\{z \in Y ; z_{n} \in N\right\}$. On $N^{\prime}$ we have $e_{\tau} \geqslant l_{n}$. It follows from (28) that

$$
\begin{aligned}
\int_{B_{n} \cap Y} \Phi\left(2 e_{\tau}\right) d P & \geqslant P\left(N^{\prime}\right) \Phi\left(2 l_{n}\right) \geqslant P(Y) P_{n}(N) \Phi\left(2 l_{n}\right) \\
& \geqslant P(Y)\left(2^{-n-4}\right)^{l_{n}} b_{n} \Phi\left(2 l_{n}\right) \geqslant 2^{n+3} P(Y) .
\end{aligned}
$$

Summation over $Y$ in $\mathscr{Y}$ gives $\int \Phi\left(2 e_{\tau}\right) d P \geqslant 2$, so $\left\|2 e_{\tau}\right\|_{\Phi} \geqslant 1$ and $\left\|e_{\tau}\right\|_{\Phi} \geqslant \frac{1}{2}$, and this concludes the proof.

\section{Proof of Theorem 11.}

LEMMA 41. If $\Psi$ fails condition $\Delta_{2}$, one can find numbers $a_{n}, b_{n}>0$, integers $k_{n}>0$ such that the following hold:

$$
\begin{gathered}
b_{n} \Psi\left(a_{n}\right) \leqslant 2^{-n}, \\
k_{n} a_{n} b_{n} \geqslant \frac{1}{4}, \\
b_{n} \Phi\left(k_{n}\right) \leqslant 1 .
\end{gathered}
$$

Proof. We can find $a_{n}$ such that $\Psi\left(a_{n}\right) \leqslant 2^{-n} \Psi\left(2 a_{n}\right)$. Let $b_{n}=1 / 2 a_{n} \psi\left(2 a_{n}\right)$. Then the equality case in Young's inequality shows that

$$
\Psi\left(2 a_{n}\right)+\Phi\left(\psi\left(2 a_{n}\right)\right)=2 a_{n} \Psi\left(2 a_{n}\right)=1 / b_{n} .
$$

It follows that $b_{n} \Psi\left(a_{n}\right) \leqslant 2^{-n} b_{n} \Psi\left(2 a_{n}\right) \leqslant 2^{-n}$. Also $b_{n} \Phi\left(\frac{1}{2} a_{n} b_{n}\right) \leqslant 1$. Since $a_{n} b_{n} \rightarrow 0$, one can suppose $2 a_{n} b_{n} \leqslant 1$ so it is enough to take for $k_{n}$ the integer part of $1 / 2 a_{n} b_{n}$.

We now proceed with the construction. Since $b_{n} \rightarrow 0$, we suppose $b_{n} \leqslant \frac{1}{2}$ for each $n$. Let $L_{n}=\left\{0, \ldots, k_{n}\right\}$. Let $M_{n}=L_{n} \backslash\{0\}$. On $L_{n}$, let $P_{n}$ be the probability given by $P_{n}(\{0\})=b_{n}, P_{n}(\{i\})=\left(1-b_{n}\right) / k_{n}$ for $i \leqslant i \leqslant k_{n}$. Let $L=\Pi L_{n}$, and $P$ be the product probability. Then $L$ is a compact metric space, and $(L, \mathscr{B}, P)$ is isomorphic to $([0,1], \mathscr{B}, \lambda)$, where $\lambda$ is Lebesgue's measure and $\mathscr{B}$ is the Borel $\sigma$-algebra.

For each $n$, let $\Sigma_{n}$ be the $\sigma$-algebra of subsets of $L$ that depend only on the first $(n-1)$ coordinates. For $i \in M_{n}$, let

$$
B_{n, i}=\left\{x \in L ; x=\left(x_{n}\right), x_{n} \in\{0, i\}\right\} .
$$


Let $\Sigma_{n, i}$ be generated by $\Sigma_{n}$ and $B_{n, i}$. We order the algebras $\Sigma_{n, i}$ by inclusion, and we denote as usual the index set by $J$, so $J=\left\{(n, i) ; n \in \mathbf{N}, i \in M_{n}\right\}$.

For each $n$, define $f_{n}$ by $f_{n}(x)=a_{n}$ if $x_{n}=0, f_{n}(x)=0$ otherwise. From (29) we see that $\int \Psi\left(f_{n}\right) d P \leqslant 2^{-n}$. On the other hand, we get that $E^{n, i}\left(f_{n}\right) \geqslant k_{n} a_{n} b_{n} \geqslant \frac{1}{4}$ on $B_{n, i}$. Since $P\left(\cup_{i \in M_{n}} B_{n, i}\right)=1-b_{n}$, we get from Lemma 28 that there is $f \in L^{\Psi}$ such that $\left(E^{t}(f)\right)$ does not converge essentially.

We show now that condition $C_{\Phi}$ holds. Let $\left(A_{t}\right)$ be an adapted family. For $t \in J$, let $A_{t}^{\prime}=A_{t} \backslash \cup_{s<t} A_{s}$. This is an adapted family, and $\cup A_{t}^{\prime} \supset A^{*}$. For each $n$, let $C_{n}=\bigcup_{i \in M_{n}} A_{n, i}^{\prime}$, so $\bigcup A_{t}^{\prime}=\bigcup C_{n}$. Let $\varepsilon>0$ and let $p$ be such that $P\left(A^{*} \backslash \bigcup_{n \leqslant p} C_{n}\right) \leqslant \varepsilon$. For $n \leqslant p$, define $\{\tau=(n, i)\}=A_{n, i}^{\prime}$ and define $\{\tau=(n, i)\}=\varnothing$ otherwise. We have $A(\tau)=\bigcup_{n \leqslant p} C_{n}$, so $P\left(A^{*} \backslash A(\tau)\right) \leqslant \varepsilon$. Since the sets $C_{n}$ are disjoint,

$$
\int \Phi\left(e_{\tau}\right) d P \leqslant \sum_{n \leqslant p} \int_{C_{n}} \Phi\left(e_{\tau}\right) d P .
$$

Let $Y$ be an atom of $\Sigma_{n}$, and $l$ be the number of $i$ in $M_{n}$ such that $A_{n, i}^{\prime} \cap Y \neq \varnothing$. For $x \in Y \cap C_{n}$, we have $e_{\tau}(x)=l-1$ if $x_{n}=0$, and zero otherwise. It follows that

$$
\int_{Y \cap C_{n}} \Phi\left(e_{\tau}\right) d P=P(Y) b_{n} \Phi(l-1) \leqslant l P(Y) b_{n} \Phi\left(k_{n}\right) / k_{n} \leqslant l P(Y) / k_{n} .
$$

On the other hand, $P\left(Y \cap C_{n}\right) \geqslant P(Y)\left(1-b_{n}\right) l / k_{n} \geqslant P(Y) l / 2 k_{n}$ so finally

$$
\int_{Y \cap C_{n}} \Phi\left(e_{\tau}\right) d P \leqslant 2 P\left(Y \cap C_{n}\right) .
$$

By summation over $Y$, we get $\int_{C_{n}} \Phi\left(e_{\tau}\right) d P \leqslant 2 P\left(C_{n}\right)$ and summation over $n$ gives $\int \Phi\left(e_{\tau}\right) d P \leqslant 2$, so $\left\|e_{\tau}\right\|_{\Phi} \leqslant 3$ and this concludes the proof.

8. Proof of Theorem 12. The details of the proof differ slightly depending upon $L^{\Psi}=L^{1}$ or $L^{\Psi} \neq L^{1}$, although the ideas are the same. We shall consider only the case $L^{\Psi} \neq L^{1}$; that is, $\Phi(u)<\infty$ for each $u$ (the case $L^{\Psi}=L^{1}$ is somewhat simpler).

Since $\Phi$ fails condition Exp, the method of Lemma 40 shows that there is a sequence $\left(a_{n}\right)$ of integers with

$$
\Phi\left(2 n a_{n}\right) 2^{-2 n-2}\left(\alpha_{n} / 2\right)^{n^{2} a_{n}} \geqslant \Phi\left(n a_{n}\right)
$$

where $\alpha_{n}=1-\left(\left(1-2^{-n-2}\right) /\left(1-2^{-n-3}\right)\right)^{1 / n}$. Let $k_{n}$ be large enough that $k_{n} \alpha_{n} \geqslant$ $2 n^{2} a_{n}$ and that $\left(1-1 / k_{n}\right)^{n} \leqslant 1-2 n / 3 k_{n}$.

Let $H_{n}=\left\{1, \ldots, k_{n}\right\}$ and let $U_{n}$ be the uniform probability on $H_{n}$. Let $M_{n}=H_{n}^{n}$, and let $Q_{n}$ be the power of $U_{n}$ on $M_{n}$. Let $N_{n}=H_{n}^{n^{2} a_{n}}$, and let $R_{n}$ be the power of $U_{n}$ on $N_{n}$. Let $L_{n}=M_{n} \cup N_{n}$. Let $b_{n}=2^{n} / \Phi\left(n a_{n}\right)$. We can assume $\Phi\left(n a_{n}\right) \geqslant 8$, so $b_{n} \leqslant 2^{-n-3}$. For $A \subset L_{n}$, let

$$
P_{n}(A)=\left(1-b_{n}\right) Q_{n}\left(A \cap M_{n}\right)+b_{n} R_{n}\left(A \cap N_{n}\right) .
$$

Let $L=\prod L_{n}$ and on $L$ let $P$ be the product of the $P_{n}$. For $i \in H_{n}$, let $D_{i}=B_{i} \cup C_{i}$, where

$$
\begin{gathered}
B_{i}=\left\{x \in M_{n} ; \exists r \leqslant n ; x(r)=i\right\}, \\
C_{i}=\left\{x \in N_{n} ; \exists r \leqslant n^{2} a_{n} ; x(r)=i\right\} .
\end{gathered}
$$


We note that

$$
Q_{n}\left(B_{i}\right)=1-\left(1-1 / k_{n}\right)^{n}, \quad R_{n}\left(C_{i}\right)=1-\left(1-1 / k_{n}\right)^{n^{2} a_{n}} .
$$

Let $L_{n}^{\prime}=\prod_{i<n} L_{i}, L_{n}^{\prime \prime}=\prod_{i>n} L_{i}$ and let $P_{n}^{\prime}$ be the product probability on $L_{n}^{\prime}$. Let $G_{n}=L_{n}^{\prime} \times H_{n}$, and $S_{n}=P_{n}^{\prime} \otimes U_{n}$. Let

$$
\mathscr{T}=\left\{T=\left(T_{n}\right) ; \forall n, T_{n} \subset G_{n}, S_{n}\left(T_{n}\right) \rightarrow 0\right\} .
$$

We order $\mathscr{T}$ by inclusion; that is, $T \leqslant T^{\prime}$ if $T_{n} \subset T_{n}^{\prime}$ for each $n$. This order makes $\mathscr{T}$ a directed set.

We can now define the index set $J$ :

$$
J=\left\{(n, f, i, T) ; n \in \mathbf{N}, f \in L_{n}^{\prime}, i \in H_{n}, T \in \mathscr{T},(f, i) \in T_{n}\right\} .
$$

The order on $J$ is defined by $(n, f, i, T) \leqslant\left(n^{\prime}, f^{\prime}, i^{\prime}, T^{\prime}\right)$ if either $n<n^{\prime}$ and $T \leqslant T^{\prime}$ or $n=n^{\prime}, T \leqslant T^{\prime}, f=f^{\prime}, i=i^{\prime}$.

We denote by $\Sigma_{n}$ the algebra of subsets of $L$ of the type $\pi_{n}^{-1}(A)$, where $A \subset L_{n}^{\prime}$ and $\pi_{n}$ is the natural projection $L \rightarrow L_{n}^{\prime}$. For $t=(n, f, i, T) \in J$, we denote by $\Sigma_{t}$ the algebra generated by $\Sigma_{n}$ and $\{f\} \times D_{i} \times L_{n}^{\prime \prime}$. It is clear that the map $t \rightarrow \Sigma_{t}$ is increasing.

We now show that condition $C_{\Phi}$ fails. For $f \in L_{n}^{\prime}, i \in H_{n}$, let $F_{f, i}=\{f\} \times D_{i} \times$ $L_{n}^{\prime \prime}$. Let us fix $m$. For $t=(n, f, i, T)$, let $A_{t}=F_{f, i}$ if $n \geqslant m$, and $A_{t}=\varnothing$ otherwise. We first show that $P\left(A^{*}\right)=1$. Let $T \in \mathscr{T}$ be fixed. It is enough to show that

$$
\lim _{n} P\left(\bigcup_{(f, i) \in T_{n}} F_{f, i}\right)=1 \text {. }
$$

Let $f \in L_{n}^{\prime}$ be fixed, let $V=\left\{i \in H_{n} ;(f, i) \in T_{n}\right\}$, and let $l=\operatorname{card} V$. We get

$$
P\left(\bigcup_{i \in V} F_{f, i}\right)=P_{n}^{\prime}(\{f\}) d_{n},
$$

where

$$
d_{n}=\left(1-b_{n}\right)\left(1-\left(1-l / k_{n}\right)^{n}\right)+b_{n}\left(1-\left(1-l / k_{n}\right)^{n^{2} a_{n}}\right) \geqslant 1-l / k_{n} .
$$

Summation over $f$ gives

$$
P\left(\bigcup_{(f, i) \in T_{n}} F_{f, i}\right) \geqslant 1-S_{n}\left(T_{n}\right),
$$

and this completes the proof that $P\left(A^{*}\right)=1$.

Now let $\tau \in I M$ with $P(A(\tau)) \geqslant \frac{1}{2}$. We show that $\left\|S_{\tau}\right\|_{\Phi} \geqslant m / 2$. For $t \in J$, let $B_{t}=\{\tau=t\} \cap A_{t}$. Since $A_{t}$ is an atom of $\Sigma_{t}$, we have either $B_{t}=A_{t}$ or $B_{t}=\varnothing$. For each $n$, let

$$
\begin{aligned}
\Gamma_{n} & =\left\{(f, i) \in L_{n}^{\prime} \times H_{n} ; \exists T \in \mathscr{T} ; B_{t}=A_{t} \text { where } t=(n, f, i, T)\right\} \\
& =\left\{(f, i) \in L_{n}^{\prime} \times H_{n} ; \exists t \in J, B_{t}=F_{f, i}\right\} .
\end{aligned}
$$

Let $\Delta_{n}=\bigcup_{(f, i) \in \Gamma_{n}} F_{f, i}$. We have $\cup B_{t}=\bigcup_{n \geqslant m} \Delta_{n}$, so there is $n \geqslant m$ such that $P\left(\Delta_{n}\right)$ $\geqslant 2^{-n-2}$. Let

$$
W=\left\{f \in L_{n}^{\prime} ; P_{n}\left(\bigcup_{(f, i) \in \Gamma_{n}} D_{i}\right) \geqslant 2^{-n-2} P_{n}^{\prime}(\{f\})\right\}
$$


From Fubini's theorem one gets $P_{n}^{\prime}(W) \geqslant 2^{-n-2}$. We fix $f \in W$. Let $V=\{i \in$ $\left.H_{n} ;(f, i) \in \Gamma_{n}\right\}$, and let $l=\operatorname{card} V$. We have

$$
\begin{aligned}
2^{-n-2} & \leqslant P_{n}\left(\bigcup_{i \in V} D_{i}\right)=\left(1-b_{n}\right)\left(1-\left(1-l / k_{n}\right)^{n}\right)+b_{n}\left(1-\left(1-l / k_{n}\right)^{n^{2} a_{n}}\right) \\
& \leqslant 1-\left(1-b_{n}\right)\left(1-l / k_{n}\right)^{n} \leqslant 1-\left(1-2^{-n-3}\right)\left(1-l / k_{n}\right)^{n} .
\end{aligned}
$$

It follows that $l / k_{n} \geqslant \alpha_{n}$. The choice of $k_{n}$ implies that $k_{n} \alpha_{n} \geqslant 2 n^{2} a_{n}$. We now estimate

$$
h_{n}=P_{n}\left(\left\{x \in L_{n} ; \sum_{i \in V} 1_{D_{i}}(x) \geqslant n^{2} a_{n}\right\}\right)
$$

We have

$$
\begin{aligned}
h_{n} & \geqslant b_{n} R_{n}\left(\left\{y \in N_{n} ; \sum_{i \in V} 1_{C_{i}}(y) \geqslant n^{2} a_{n}\right\}\right) \\
& =b_{n}\left(\frac{l}{k_{n}} \frac{l-1}{k_{n}} \cdots \frac{l-n^{2} a_{n}+1}{k_{n}}\right) \geqslant b_{n}\left(\frac{l}{2 k_{n}}\right)^{n^{2} a_{n}} \geqslant b_{n}\left(\alpha_{n} / 2\right)^{n^{2} a_{n}} .
\end{aligned}
$$

It follows that if $G=\{f\} \times L_{n-1}^{\prime \prime}$, we have

$$
\int_{G} \Phi\left(\frac{2}{n} \sum_{i \in V} 1_{D_{i}}\right) d P \geqslant b_{n}\left(\alpha_{n} / 2\right)^{n^{2} a_{n}} \Phi\left(2 n a_{n}\right) P_{n}^{\prime}(\{f\}) .
$$

Summation for $f \in W$ gives

$$
\begin{aligned}
\int \Phi\left(\frac{2}{n} S_{\tau}\right) d P & \geqslant \int \Phi\left(\frac{2}{n} \sum_{(f, i) \in \Gamma_{n}} 1_{F_{f, i}}\right) d P \\
& \geqslant 2^{-n-2} b_{n}\left(\alpha_{n} / 2\right)^{n^{2} a_{n}} \Phi\left(2 n a_{n}\right) \geqslant 1 .
\end{aligned}
$$

So $\left\|S_{\tau}\right\|_{\Phi} \geqslant n / 2 \geqslant m / 2$.

We now start proving that condition $F V_{\Phi}$ holds.

Lemma 42. Let $\left(A_{t}\right)_{t \in J}$ be an adapted family such that, for each $t=(n, f, i, T), A_{t}$ is $\Sigma_{n}$-measurable. Then there is a disjoint adapted family $\left(B_{t}\right)_{t \in J}$, with $B_{t} \subset A_{t}$ and $P\left(\cup B_{t}\right) \geqslant P\left(\bigcup A_{t}\right)$.

Proof. This is actually almost obvious. Let

$$
J_{n}=\left\{(n, f, i, T) \in J ; f \in L_{n}^{\prime}, i \in I_{n}, T \in \mathscr{T}\right\} .
$$

Let $\Delta_{n}^{\prime}=\bigcup_{t \in J_{n}} A_{t}, \Delta_{n}=\Delta_{n}^{\prime} \backslash \bigcup_{m<n} \Delta_{m}^{\prime}$. We have $\bigcup \Delta_{n}=\bigcup A_{t}$ and the sets $\Delta_{n}$ are disjoint. For $t \in J_{n}$, let $A_{t}^{\prime}=A_{t} \cap \Delta_{n} \in \Sigma_{n}$, so $\bigcup A_{t}^{\prime}=\bigcup A_{t}$. We can find a disjoint family $\left(B_{t}\right)_{t \in J_{n}}, B_{t} \in \Sigma_{n}, B_{t} \in A_{t}^{\prime}$ with $\bigcup_{J_{n}} B_{t}=\bigcup_{J_{n}} A_{t}$, so the family $\left(B_{t}\right)_{t \in J}$ is disjoint and has the same union as $\left(A_{t}\right)$.

LEMMA 43. Let $\left(A_{t}\right)$ be an adapted family such that for $t=(n, f, i, T) \in J$, we have $A_{t} \subset\{f\} \times D_{i}^{c} \times L_{n}^{\prime \prime}$, where $D_{i}^{c}=L_{n} \backslash D_{i}$. Then for each $\varepsilon>0$ there is a disjoint adapted family $\left(B_{t}\right), B_{t} \subset A_{t}$, with $P\left(\cup B_{t}\right) \geqslant P\left(A^{*}\right)-\varepsilon$. 
Proof. Let $m$ be such that for $n \geqslant m$ and $i \in H_{n}$ we have $P_{n}\left(D_{i}\right) \leqslant \varepsilon$. For $t=(n, f, i, T) \in J, n \geqslant m$, let $A_{t}^{\prime}=\{f\} \times L_{n-1}^{\prime \prime}$ if $A_{t}=\{f\} \times D_{i}^{c} \times L_{n}^{\prime \prime}$, and $A_{t}^{\prime}=$ $\varnothing$ if $A_{t}=\varnothing$ (note that $\{f\} \times D_{i}^{c} \times L_{n}^{\prime \prime}$ is an atom of $\Sigma_{t}$, so no other case is possible). Now $A_{t}^{\prime} \in \Sigma_{n}$, and $P\left(\cup A_{t}^{\prime}\right) \geqslant P\left(A^{*}\right)$. Lemma 42 gives a disjoint adapted family $B_{t}^{\prime} \subset A_{t}^{\prime}$ with $P\left(\cup B_{t}^{\prime}\right)=P\left(\cup A_{t}^{\prime}\right)$, and the proof of Lemma 42 shows that, for $t=(n, f, i, T), B_{t}^{\prime}$ is actually $\Sigma_{n}$-measurable, so either $B_{t}^{\prime}=\varnothing$ or $B_{t}^{\prime}=\{f\} \times L_{n-1}^{\prime \prime}$. Define $B_{t}=A_{t}$ whenever $B_{t}^{\prime} \neq \varnothing$. Since $B_{t} \subset B_{t}^{\prime}$, the family $\left(B_{t}\right)$ is disjoint. Also, since $P\left(B_{t}\right) \geqslant(1-\varepsilon) P\left(B_{t}^{\prime}\right)$, we have $P\left(\bigcup B_{t}\right) \geqslant(1-\varepsilon) P\left(\bigcup A_{t}^{\prime}\right) \geqslant(1-\varepsilon) P\left(A^{*}\right)$, and the proof is complete.

We now come to the main argument.

Proposition 44. Assume that for each $t=(n, f, i, T) \in J$, either $A_{t}=\varnothing$ or $A_{t}=F_{f, i}$. Then for each $m>0$ and each $\varepsilon>0$ there are numbers $\gamma_{t}$ such that if $\eta=\sum \gamma_{t} 1_{A_{t}}$, we have $\int_{\{\eta>1\}} \Phi(m \eta) d P \leqslant 1$ and $P\left(A^{*} \cap\{\eta \geqslant 1\}\right) \geqslant P\left(A^{*}\right)-\varepsilon$.

Proof. First step. For each $n$ let

$$
\Gamma_{n}=\left\{(f, i) \in L_{n}^{\prime} \times H_{n} ; \exists T \in \mathscr{T}, A_{t}=F_{f, i} \text { for } t=(n, f, i, T)\right\} .
$$

Let $\beta>0$. Let

$$
W_{n}(\beta)=\left\{f \in L_{n}^{\prime} ; \operatorname{card}\left\{i \in H_{n} ;(f, i) \in \Gamma_{n}\right\} \geqslant \beta k_{n}\right\} .
$$

Let $Z_{n}(\beta)=\bigcup\left\{F_{f, i} ;(f, i) \in W_{n}(\beta)\right\}$. Let $Z^{*}(\beta)=\limsup Z_{n}(\beta)$. When $\beta$ decreases, $Z^{*}(\beta)$ increases. We show that there is a $\beta>0$ such that $P\left(A^{*} \backslash Z^{*}(\beta)\right)<$ $\varepsilon / 2$.

Otherwise, if $Z^{*}=\bigcup_{\beta>0} Z^{*}(\beta)$, we have $P\left(A^{*} \backslash Z^{*}\right) \geqslant \varepsilon / 2$. For each $k>0$, let $n_{k}$ be such that

$$
P\left(\bigcup_{n \geqslant n_{k}} Z_{n}\left(2^{-k}\right) \backslash Z^{*}\left(2^{-k}\right)\right) \leqslant \varepsilon 2^{-k-2}
$$

Let

$$
Z^{\prime}=\bigcup_{k} \bigcup_{n_{k} \leqslant n<n_{k+1}} Z_{n}\left(2^{-k}\right)
$$

We have $P\left(A^{*} \backslash Z^{\prime}\right) \geqslant \varepsilon / 4>0$. For each $n$, let $k(n)$ be the unique $k$ with $n_{k} \leqslant n<$ $n_{k+1}$. Let

$$
T_{n}=\left\{(f, i) \in L_{n}^{\prime} \times H_{n} ;(f, i) \in \Gamma_{n}, f \notin W_{n}\left(2^{-k(n)}\right)\right\} .
$$

Then $S_{n}\left(T_{n}\right) \leqslant 2^{-k(n)}$, so $S_{n}\left(T_{n}\right) \rightarrow 0$ and $T=\left(T_{n}\right) \in \mathscr{T}$.

Consider

$$
A^{\prime}=\bigcup\left\{A_{t} ; t=\left(n, f, i, T^{\prime}\right) ; T^{\prime} \geqslant T\right\} .
$$

Let $(f, i) \in L_{n}^{\prime} \times H_{n}$ such that there exists $T^{\prime} \geqslant T$ with $A_{t}=F_{f, i}$, for $t=$ $\left(n, f, i, T^{\prime}\right)$. Since $(f, i) \in T_{n}^{\prime}$, we have $(f, i) \in T_{n}$. Since $(f, i) \in \Gamma_{n}$, we have $f \in W_{n}\left(2^{-k(n)}\right)$, so $F_{f, i} \subset Z_{n}\left(2^{-k(n)}\right)$. It follows that $A^{\prime} \subset Z^{\prime}$. This contradicts the fact that $P\left(A^{*} \backslash Z^{\prime}\right)>0$, and proves the claim. We fix $\beta$ with $P\left(A^{*} \backslash Z^{*}(\beta)\right)<\varepsilon / 2$. 
Second step. Consider a set $V \subset H_{n}$ with $l=\operatorname{card} V \geqslant \beta k_{n}$. Consider the function $\eta=(1 / n m) \sum_{i \in V} 1_{D_{i}}$. We have $\|\eta\|_{1}=(l / n m) P_{n}\left(D_{1}\right)$, so (32) shows that there is $n_{0}$ such that, if $n>n_{0}$, we have $\|\eta\|_{1} \geqslant \beta / 2 m$. Since any collection of $n+1$ sets $B_{i}$ has empty intersection, we have $\eta \leqslant 1 / m \leqslant 1$ on $M_{n}$. Similarly, $\eta \leqslant n a_{n} / m$, so we have

$$
\int_{\{\eta>1\}} \Phi(m \eta) d P \leqslant b_{n} \Phi\left(n a_{n}\right) \leqslant 2^{-n} .
$$

Third step. Let us fix $p>n_{0}$ and let $K$ be an atom of $\Sigma_{p}$. Let $\gamma>0$. We show that we can find a finite set $I \subset J$ and for $t \in I$ numbers $\gamma_{t}$ such that, if $\eta=\sum_{I} \gamma_{t} 1_{A_{t} \cap K}$, we have $\|\eta\|_{1} \geqslant(\beta / 4 m) P\left(Z^{*}(\beta) \cap K\right)$ and $\int_{\{\eta>1\}} \Phi(m \eta) d P \leqslant \gamma$.

Let $p_{0}>p$ with $2^{-p_{0}} \leqslant \gamma$. For $n \geqslant p_{0}$, we define

$$
Z_{n}^{\prime \prime}=\bigcup\left\{\{f\} \times L_{n-1}^{\prime \prime} ; f \in W_{n}(\beta)\right\} \cap K .
$$

$Z_{n}^{\prime \prime}$ belongs to $\Sigma_{n}$, and $Z_{n}^{\prime \prime} \supset Z_{n}(\beta) \cap K$,

$$
\bigcup_{n \geqslant p_{0}} Z_{n}^{\prime \prime} \supset \bigcup_{n \geqslant p_{0}} Z_{n}(\beta) \cap K \supset Z^{*}(\beta) \cap K .
$$

Let $Z_{n}^{\prime}=Z_{n}^{\prime \prime} \backslash \bigcup_{q<n} Z_{q}^{\prime \prime}$. The sets $Z_{n}^{\prime}$ are disjoint. Let $\pi_{n}$ be the natural projection of $L$ on $L_{n}^{\prime}$, and let $V_{n}=\pi_{n}\left(Z_{n}^{\prime}\right)$.

Let $p^{\prime}$ be such that

$$
P\left(\bigcup_{p_{0} \leqslant n \leqslant p^{\prime}} Z_{n}^{\prime \prime}\right) \geqslant P\left(Z^{*}(\beta) \cap K\right) / 2 .
$$

Define $\eta=\Sigma(1 / n m) 1_{F_{f, i}}$ where the summation is for $p_{0} \leqslant n \leqslant p^{\prime},(f, i) \in \Gamma_{n}$. For $f \in \mathscr{V}_{n}$, if $K^{\prime}=\pi_{n}^{-1}(\{f\})$, the second step shows that

$$
\left\|\eta 1_{K^{\prime}}\right\|_{1} \geqslant \beta P\left(K^{\prime}\right) / 2 m, \quad \int_{K^{\prime} \cap\{\eta>1\}} \Phi(m \eta) d P \leqslant \gamma P\left(K^{\prime}\right),
$$

and the result follows by summation over $f \in V_{n}, p \leqslant n \leqslant p^{\prime}$.

Fourth step. By induction over $k$ we show that if $K$ is an atom of $\Sigma_{p}, p \geqslant n_{0}$, there is a finite set $i$, and for $t \in I$ a $\gamma_{t}$ such that if $\eta=\Sigma_{I} \gamma_{t} 1_{A_{t}}$, we have

$$
\begin{gathered}
\|\eta\|_{1} \geqslant\left(1-(1-\beta / 4 m)^{k}\right) P\left(Z^{*}(\beta) \cap K\right), \\
\int_{\{n>1\}} \Phi(m \eta) d P \leqslant\left(1-(1-\beta / 4 m)^{k}\right) P\left(Z^{*}(\beta) \cap K\right) .
\end{gathered}
$$

The proof of the proposition will follow by taking $k$ large enough that

$$
(1-\varepsilon / 2)(1-\beta / 4 m)^{k}>1-\varepsilon
$$

and by summation over the atoms of $\Sigma_{n_{0}}$.

The case $k=1$ has been proved in the third step. Suppose the result has been proved up to $k$. Let $K$ be an atom of $\Sigma_{p}$, and let $\eta$ satisfy (34) and (35). We can suppose $\|\eta\|_{1}<P\left(Z^{*}(\beta) \cap K\right)$. Let $q$ be such that $\eta$ is $\Sigma_{q}$-measurable. Let us fix an atom $F$ of $\Sigma_{q}$. Let

$$
d_{F}=(\beta / 4 m)(1-\beta / 4 m)^{k} P\left(Z^{*}(\beta) \cap F\right) .
$$


The third step gives a function $\eta^{\prime}$ such that

$$
\begin{gathered}
\left\|\eta^{\prime}\right\|_{1} \geqslant(\beta / 4 m) P\left(Z^{*}(\beta) \cap F\right), \\
\int_{\left\{\eta^{\prime}>1\right\}} \Phi\left(m \eta^{\prime}\right) d P \leqslant d_{F} .
\end{gathered}
$$

Define $\eta_{F}=\eta 1_{F}$ if $\eta \geqslant 1$ on $F$ and $\eta_{F}=\left(\eta+\eta^{\prime}(1-\eta)\right) 1_{F}$ if $\eta<1$ on $F$. If $a_{F}$ denotes the (constant) value of $\eta$ on $F$, we have

$$
\left\|\eta_{F}\right\|_{1}=a_{F} P(F)+\left(1-a_{F}\right)\|\eta\|_{1} \geqslant a_{F} P(F)+\left(1-a_{F}\right)(\beta / 4 m) P\left(Z^{*}(\beta) \cap F\right),
$$

so

$$
P\left(Z^{*}(\beta) \cap F\right)-\left\|\eta_{F}\right\|_{1} \leqslant(1-\beta / 4 m)\left(P\left(Z^{*}(\beta) \cap F\right)-a_{F} P(F)\right) .
$$

If $\eta \leqslant 1$ we have $\eta_{F}>1 \Rightarrow \eta^{\prime}>1$, so

$$
\int_{\left\{\eta_{F}>1\right\}} \Phi\left(m \eta_{F}\right) d P \leqslant \int_{\left\{\eta^{\prime}>1\right\}} \Phi\left(m\left(a_{F}+\eta^{\prime}\left(1-a_{F}\right)\right)\right) d P \leqslant \int_{\left\{\eta^{\prime}>1\right\}} \Phi\left(m \eta^{\prime}\right) d P .
$$

Define $\eta_{1}=\sum \eta_{F}$, where the summation is taken for the atoms $F$ on $\Sigma_{c}$ contained in $F$. Summation of (39) gives

$$
\begin{aligned}
\int_{\left\{\eta_{1}>1\right\}} \Phi\left(m \eta_{1}\right) d P & \leqslant \int_{\{\eta>1\}} \Phi(m \eta) d P+\sum d_{F} \\
& \leqslant\left(1-(1-\beta / 4 m)^{k+1}\right) P\left(Z^{*}(\beta) \cap K\right) .
\end{aligned}
$$

We note that (38) remains true if $\eta \geqslant 1$ on $F$, since in that case $\left\|\eta_{F}\right\|_{1}=a_{F} P(F)$ $>P\left(Z^{*}(\beta) \cap F\right)$. Summation over the $F \subset K$ gives

$$
P\left(Z^{*}(\beta) \cap F\right)-\left\|\eta_{1}\right\|_{1} \leqslant(1-\beta / 4 m)\left(P\left(Z^{*}(\beta) \cap F\right)-\|\eta\|_{1}\right)
$$

and this implies

$$
\left\|\eta_{1}\right\|_{1} \geqslant\left(1-(1-\beta / 4 m)^{k+1}\right) P\left(Z^{*}(\beta) \cap F\right) .
$$

The proposition is proved.

We now complete the proof that condition $F V_{\Phi}$ holds. Let $\left(A_{t}\right)$ be an adapted family of sets. If $t=(n, f, i, T)$, let

$$
A_{t}^{1}=A_{t} \backslash\left(\pi_{n}^{-1}(f)\right), \quad A_{t}^{2}=A_{t} \cap\left(\{f\} \times D_{i}^{c} \times L_{n}^{\prime \prime}\right), \quad A_{t}^{3}=A_{t} \cap F_{n, i} .
$$

Let $\varepsilon>0$. It follows from Lemma 42 that there exists $\tau_{1} \in I M$ with $\left\{\tau_{1}=t\right\} \subset A_{t}$ for each $t, P\left(A^{1^{*}} \backslash A^{1}\left(\tau_{1}\right)\right) \leqslant \varepsilon / 3$ and $e_{\tau_{1}}=0$. Let $t_{1}$ be such that $\tau_{1} \leqslant t_{1}$. For $t \geqslant t_{1}$, let $A_{t}^{\prime}=A_{t}^{2} \backslash A^{1}\left(\tau_{1}\right)$ and let $A_{t}^{\prime}=\varnothing$ otherwise. From Lemma 43 there is $\tau_{2} \in I M$, with $\left\{\tau_{2}=t\right\} \subset A_{t}$ for each $t, P\left(A^{\prime *} \backslash A^{\prime}\left(\tau_{2}\right)\right) \leqslant \varepsilon / 3$ and $e_{\tau_{2}}=0$. Let $t_{2}$ with $\tau_{2} \leqslant t_{2}$. For $t \geqslant t_{2}$, let $A_{t}^{\prime \prime}=A_{t}^{3} \backslash\left(A^{1}\left(\tau_{1}\right) \cup A^{2}\left(\tau_{2}\right)\right)$ and let $A_{t}^{\prime \prime}=\varnothing$ otherwise. From Lemma 44 there are numbers $\gamma_{t}$ such that, if $\eta=\sum \gamma_{t} A_{t}^{\prime \prime}$, then $\|\eta\|_{1} \geqslant P\left(A^{\prime \prime *}\right)$ $-\varepsilon / 3$ and $\int_{\{\eta>1\}} \Phi(\eta / \varepsilon) d P \leqslant 1$. Define now $\eta^{\prime}=\eta+\sum 1_{\left\{\tau_{1}=t\right\}}+\sum 1_{\left\{\tau_{2}=t\right\}}$. Since $A^{*} \subset A^{1^{*}} \cup A^{2^{*}} \cup A^{3^{*}}$, we have $\left\|\eta^{\prime}\right\|_{1} \geqslant P\left(A^{*}\right)-\varepsilon$ and $\int_{\left\{\eta^{\prime}>1\right\}} \Phi\left(\eta^{\prime} / \varepsilon\right) d P \leqslant 1$, so $\left\|\eta^{\prime}-\eta^{\prime} \wedge 1\right\|_{\Phi} \leqslant \varepsilon$. This completes the proof of Theorem 12 . 
9. Proof of Theorem 13. Let $k_{n}=2^{n}$ and $L_{n}=\left\{0, \ldots, k_{n}\right\}$. Let $P_{n}$ be the uniform probability on $L_{n}$. Let $L=\Pi L_{n}$ and let $P$ be the product probability on $L$. Let $L_{n}^{\prime}=\prod_{i<n} L_{i}$. Let $\pi_{n}$ be the natural projection of $L$ on $L_{n}^{\prime}$. For $x \in L_{n}^{\prime}$, let

$$
H_{n}(x)=\left\{y=\left(y_{n}\right) \in L ; \pi_{n}(y)=x, y_{n}=0\right\}
$$

and let $H_{n}=\bigcup_{x \in L_{n}^{\prime}} H_{n}(x)$, so $H_{n}=\left\{y \in L ; y_{n}=0\right\}$. Let $G_{n}=\bigcup_{n \leqslant m} H_{m}$. We note that $P\left(H_{n}\right)=1 /\left(k_{n}+1\right)$, so $P\left(G_{n}\right) \rightarrow 0$. For $x \in L_{n}^{\prime}$, let $\tilde{x}=\pi_{n}^{-1}(x)$.

Let $\omega_{1}$ denote the first uncountable ordinal. Continuum Hypothesis means that $\omega_{1}$ has the power of continuum. So, we can find an enumeration $\left(f_{\alpha}, \eta_{\alpha}\right)_{\alpha<\omega_{1}}$ of all the couples $(f, n)$, where $f \in L^{1}(P), f \geqslant 0$, and $n \in \mathbf{N}$. By induction over $\alpha<\omega_{1}$, we construct a sequence of Borel sets $B_{\alpha}$ such that the following hold:

(40) $B_{\alpha} \supset L \backslash G_{n_{\alpha}}$;

(41) for each $n$, each $x \in L_{n+1}^{\prime}$, either $B_{\alpha} \supset \tilde{x} \backslash G_{n}$ or $B_{\alpha} \cap \tilde{x} \subset G_{n}$;

(42) whenever $R$ is a finite subset of $\left[0, \omega_{1}\left[\right.\right.$, the set $T(R)=\bigcap_{\alpha \in R} B_{\alpha}$ meets every set $H_{n}(x)$ for $n \in \mathbf{N}, x \in L_{n}^{\prime}$, in a set with nonempty interior;

(43) if $n>n_{\alpha}, x \in L_{n}^{\prime}, x_{i} \neq 0$ for $i<n-1, x_{n-1}=0$, then

$$
\int_{\tilde{x} \cap B_{\alpha}} f_{\alpha} d P \leqslant 2^{-n} P(\tilde{x}) .
$$

We first construct $B_{0}$. We can enumerate the sets $H_{n}(x)$ as $Z_{l}=H_{n(l)}(x(l))$. By induction over $l$, we construct an increasing sequence $(p(l))$ with $p(1)>n_{0}$, and a sequence $y(l) \in L_{p(l)}^{\prime}$ such that the following properties hold:

(44) $p(l)>n(l)+2$;

(45) the component of $y(l)$ of index $p(l)-1$ is zero and $\tilde{y}(l) \subset Z_{l}$;

(46) $\int_{\tilde{y}(l)} f_{0} d P \leqslant 2^{-l-n(l)} P\left(Z_{l}\right)$.

The construction is easy. There are $K=\Pi\left(k_{i}+1\right)$ (where the product is for $n(l)<i<p(l)-1)$ possible choices of $y(l)$ satisyfing (45), and the corresponding sets $\tilde{y}(l)$ are disjoint. So if $p(l)$ is large enough that $K 2^{-l-n(l)} P\left(Z_{l}\right) \geqslant\left\|f_{0}\right\|_{1}$, we can find one of them for which (46) holds.

We now prove (40). Let $B_{0}=L \backslash\left(G_{n_{0}} \backslash \bigcup_{l} \tilde{y}(l)\right)$. Since the component of $y(l)$ of index $p(l)-1$ is zero, we have $\tilde{y}(l) \subset G_{n_{0}}$ for each $l$, so (40) holds, and $B_{0}=$ $\left(L \backslash G_{n_{0}}\right) \cup \cup_{l} \tilde{y}(l)$.

We now prove (41). Let us fix $n$ and $x \in L_{n+1}^{\prime}$. Suppose that we have $\tilde{x} \cap B_{0} \neq \varnothing$. If $\tilde{x} \not \subset G_{n_{0}}$, the components of $\tilde{x}$ of index between $n_{0}$ and $n$ are nonzero, so $\tilde{x} \cap G_{n_{0}}=\tilde{x} \cap G_{n_{0}}$, so $\tilde{x} \backslash G_{n}=\tilde{x} \backslash G_{n_{0}}$, and $B_{\alpha} \cap \tilde{x} \supset \tilde{x} \backslash G_{n}$ from (40). If $\tilde{x} \subset G_{n_{0}}$, then $\tilde{x} \cap B_{0}=\tilde{x} \cap \bigcup_{l} \tilde{y}(l)$. If for some $l$ we have $\tilde{x} \subset \tilde{y}(l)$, then $\tilde{x} \backslash G_{n} \subset \tilde{x} \subset B_{0}$. Otherwise let us fix $l$ with $\tilde{y}(l) \cap \tilde{x} \neq \varnothing$. Then $\tilde{y}(l) \subset \tilde{x}$ and $p(l)>n$. Since the last component of $y$ is zero, we have $\tilde{y}(l) \subset G_{n}$, so $B \cap \tilde{x} \subset G_{n}$ in that case. This proves (41).

We now prove (43). Let $n>n_{0}, x \in L_{n}^{\prime}$ with $x_{i} \neq 0$ for $i<n-1$ and $x_{n-1}=0$. We have $\tilde{x} \subset G_{n_{0}}$. We have $\tilde{x} \subset G_{n_{0}}$. Fix $l$. Suppose $\tilde{x} \cap \tilde{y}(l) \neq \varnothing$. We cannot have $p(l) \leqslant n$, since $y(l)$ has at least two components of index $\leqslant p(l)-1$ which are zero (i.e. those of index $n(l)$ and $p(l))$ while $x$ has at most one. So if $\tilde{y}(l) \cap \tilde{x} \neq \varnothing$, we have $p(l)>n$. Since the component of $\tilde{y}$ of rank $n(l)$ is zero, we have $n(l) \geqslant n-1$, 
so $P\left(Z_{l}\right) \leqslant P(\tilde{x})$. Now (46) implies that

$$
\int_{\tilde{x} \cap \tilde{y}(l)} f_{0} d P \leqslant 2^{-n-l} P\left(Z_{l}\right) \leqslant 2^{-n-l} P(\tilde{x})
$$

and this implies (43).

We now construct the sets $B_{\alpha}$ by induction. Suppose that the sets $B_{\alpha}$ have been constructed whenever $\alpha<\beta$. We can enumerate as

$$
U_{l}=T\left(R_{l}\right) \cap H_{n(l)}(x(l))
$$

the sets $T(R) \cap H_{n}(x)$ where $R$ is a finite subset of [0, $\beta$ [, and $x \in L_{n}^{\prime}$. Let $Z_{l}=H_{n(l)}(x(l))$. By induction over $l$, we construct an increasing sequence $(p(l))$ with $p(1)>n_{\beta}$ and a sequence $y(l) \in L_{p(l)}^{\prime}$ such that (40) holds, together with the following:

(47) the component of $y(l)$ of index $p(l)-1$ is zero and $\tilde{y}(l) \subset U_{l}, P(\tilde{y}(l)) \leqslant$ $2^{-l-1} P\left(U_{l}\right)$;

(48) $\int_{\tilde{y}(l)} f_{\beta} d P \leqslant 2^{-l-n(l)} P\left(Z_{l}\right)$.

The construction is very similar to the construction in the case $\alpha=0$, so we leave it to the reader. We set $B_{\beta}=L \backslash\left(G_{n_{\beta}} \backslash \cup \tilde{y}(l)\right)$. To check that (44)-(46) hold, one proceeds as in the case $\alpha=0$. We now check (42). Let $R$ be a finite subset of [0, $\beta$, and fix $n \in \mathbf{N}, x \in L_{n}^{\prime}$. We now show that $T(R) \cap H_{n}(x) \cap B_{\beta}$ has nonempty interior. There exists $n^{\prime} \geqslant n_{\beta}$, and $x^{\prime} \in L_{n^{\prime}}^{\prime}$ with $H_{n^{\prime}}\left(x^{\prime}\right) \subset H_{n}(x)$. So we can actually suppose $n \geqslant n_{\beta}$, so we have $H_{n}(x) \subset G_{n_{\beta}}$. By construction there is $l$ with $\tilde{y}(l) \subset T(R) \cap H_{n}(x)$, so $\tilde{y}(l) \subset T(R) \cap H_{n}(x) \cap B_{\beta}$. This completes the construction.

Let $M_{n}=\left\{1, \ldots, k_{n}\right\}$. For $i \in M_{n}$, let $C_{n, i}=\left\{y \in L ; y_{n} \in\{0, i\}\right\}$. Let $\Sigma_{n}$ be the algebra of subsets of $L$ that depend only on the coordinates of rank $\leqslant n-1$. Let $S$ be the set of finite subsets of $\left[0, \omega_{1}[\right.$, ordered by inclusion. The index set $J$ is given by

$$
J=\left\{(n, i, R): i \in M_{n}, R \in S\right\} .
$$

We order it by $(n, i, R) \leqslant\left(n^{\prime}, i^{\prime}, R^{\prime}\right)$ if either $n<n^{\prime}, R \subset R^{\prime}$ or $n=n^{\prime}, i=i^{\prime}$, $R \subset R^{\prime}$. This order makes $J$ a directed set. For $t \in J, t=(n, i, R)$, let $\Sigma_{t}$ be the algebra generated by $\Sigma_{n}, C_{n, i}$, and the sets $B_{\alpha}$ for $\alpha \in R$. The map $t \rightarrow \Sigma_{t}$ is increasing. Denote by $\Xi$ the union of the algebras $\Sigma_{t}$. For $x \in L_{n}^{\prime}, q \in \mathbf{N}$, let

$$
F_{n, q}(x)=\left\{y \in L ; \pi_{n}(y)=x, y_{i}=0, \forall n \leqslant i \leqslant n+q\right\} .
$$

Let $a_{n}=2^{-n} /$ card $L_{n}^{\prime}$. From (42) there exists a unique positive finitely additive measure $\mu_{n, x}$ such that $\mu_{n, x}(L)=a_{n}$ and

$$
\forall \alpha<\omega_{1}, \quad \mu_{n, x}\left(B_{\alpha}\right)=a_{n}, \quad \forall q, \mu_{n, x}\left(F_{n, q}(x)\right)=a_{n} .
$$

Let $\mu=\sum \mu_{n, x}$, where the summation is over $n$ and $x \in L_{n}^{\prime}$. Note that $\mu(L)=1$. For $t \in J$, we define $X=\left(X_{t}\right)$ by $X_{t}=\mu(A) / P(A)$ on $A$ for each atom $A$ of $\Sigma_{t}$. This is a positive martingale, and $\left\|X_{t}\right\|_{1}=1$ for each $t$. We show that it does not converge. It is clear that $\mu$ is singular; that is, for each $\varepsilon>0$ there is $t \in J$ and $A \in \Sigma_{t}$ with $P(A) \geqslant 1-\varepsilon, \mu(A) \leqslant \varepsilon$. This shows that $X_{*}=0$. 
Now let $n \in \mathbf{N}$, and $R$ be a finite subset of [0, $\omega_{1}\left[\right.$. Let $y \in L$ with $y \notin G_{1}$. Then from (40) $y$ belongs to $T(R)$. Let $z=\pi_{n}(y) \in L_{n}^{\prime}$, and let $i=y_{n}$. Then $y \in A=\tilde{z} \cap$ $C_{n, i} \cap T(R)$ and $A$ is an atom of $\Sigma_{t}$, for $t=(n, i, R)$. We have

$$
\mu(A) \geqslant \mu_{n, z}(A) \geqslant \mu_{n, z}\left(T(R) \cap H_{n}(z)\right)=a_{n} .
$$

On the other hand,

$$
m(A) \leqslant m\left(C_{n, i}\right) \leqslant 2 / k_{n} \text { card } L_{n}^{\prime}=2^{-n+1} / \operatorname{card} L_{n}^{\prime}=2 a_{n},
$$

so $X_{t}(y) \geqslant \frac{1}{2}$. This shows that $X^{*} \geqslant \frac{1}{2}$ on $L \backslash G_{1}$. Since $P\left(L \backslash G_{1}\right)>0$, we have $X^{*} \neq X_{*}$, so $\left(X_{t}\right)$ does not converge essentially.

Now let $f \in L^{1}(P)$. We show that $X_{t}=\left(E^{t}(f)\right)$ converges. By approximation, it is enough to show that $P\left(X^{*} \geqslant \lambda\right) \leqslant\|f\|_{1} / \lambda$ when $f \geqslant 0, \lambda>0$.

Let $g=\operatorname{Sup}_{n}\left(E\left(f \mid \Sigma_{n}\right)\right)$. We know that $P(g>\lambda) \leqslant\|f\|_{1} / \lambda$ since the sequence $\Sigma_{n}$ is increasing. Let $m>0$. Let $\alpha<\omega_{1}$ with $(f, m)=\left(f_{\alpha}, n_{\alpha}\right)$. We show that if $(m, 1,\{\alpha\}) \leqslant t=(n, i, R)$, then $X_{t} \leqslant c_{m} g+d_{m}$ on $L \backslash G_{m}$, where $c_{m}, d_{m}$ depend only on $m$ and $c_{m} \rightarrow 1, d_{m} \rightarrow 0$. This implies $X^{*} \leqslant g$ on $L$, and concludes the proof. Let $y \in L \backslash G_{m}$. Let $x=\pi_{m}(y)$. It follows from (41) that, for each $\beta \in R$, either $\tilde{x} \backslash G_{m} \subset B_{\beta}$ or $\left(\tilde{x} \backslash G_{m}\right) \cap B_{\beta}=\varnothing$. Let $i=y_{m}$. It follows that the atom $A$ of $\Sigma_{t}$ that contains $y$ contains $\left(\tilde{x} \backslash G_{m}\right) \cap C_{m, i}$, so if $z=\pi_{m+1}(y), A$ contains $\tilde{z} \backslash G_{m}$. Easy computation shows $P\left(\tilde{z} \backslash G_{m}\right) \geqslant P(\tilde{z}) r_{m}$, where $r_{m}=\Pi_{i \geqslant m}\left(1-2^{-i}\right)$, so $P(A) \geqslant$ $P(\tilde{z}) r_{n}$.

Since $y \notin G_{m}$, we have $y \in B_{\alpha}$ from (40), so $A \subset \tilde{z} \cup\left(H_{m}(x) \cap B_{\alpha}\right)$. From (43) it follows that

$$
\int_{H_{n}(x) \cap B_{\alpha}} f_{\alpha} d P \leqslant 2^{-m} P\left(H_{m}(x)\right)=2^{-m} P(\tilde{z}) .
$$

So we have

$$
\begin{aligned}
X_{t}(y) & \leqslant \int_{A} f_{\alpha} d P / P(A) \leqslant\left(\int_{\tilde{z}} f_{\alpha} d P\right) /\left(r_{m} P(\tilde{z})\right)+2^{-m} / r_{m} \\
& \leqslant g(y) / r_{m}+2^{-m / r_{m}} .
\end{aligned}
$$

The proof is complete.

10. Proof of Theorem 21. We shall perform the construction in the case $L^{\Psi} \neq L^{1}$; that is, $\Phi<\infty$ everywhere. The details in the case $L^{\Psi}=L^{1}$ are somewhat simpler.

Since $\Phi$ fails condition Exp, Lemma 40 shows that there is a sequence $\left(l_{n}\right)$ of integers such that

$$
\Phi\left(2 l_{n}\right) \geqslant 2^{2 n}\left(2^{2 n+1}\right)^{n l_{n}} \Phi\left(l_{n}\right) .
$$

We can assume $\Phi\left(l_{n}\right) \geqslant 2$. Let $b_{n}=2^{-n} / \Phi\left(l_{n}\right), k_{n}=n 2^{n+1} l_{n}$. Let

$$
H_{n}=\left\{1, \ldots, k_{n}\right\} \times\left\{1, \ldots, 2^{n}\right\} .
$$

Let $U_{n}$ be the uniform probability on $H_{n}$. Let $M_{n}=H_{n}^{n l_{n}}$, and let $Q_{n}$ be the power of $U_{n}$ on $M_{n}$. Let $L_{n}=H_{n} \cup M_{n}$, and on $L_{n}$, let $P_{n}$ be given by

$$
P_{n}(A)=\left(1-b_{n}\right) U_{n}\left(A \cap H_{n}\right)+b_{n} Q_{n}\left(A \cap M_{n}\right) \text {. }
$$


Let $L=\Pi L_{n}$, and $\mu$ be the product measure. Let $F_{n}=\left\{x \in L ; x_{n} \in H_{n}\right\}$. For $x=\left(x_{m}\right) \in F_{n}$, write $x_{n}=\left(p_{n}(x), q_{n}(x)\right)$ where $P_{n}(x) \in\left\{1, \ldots, k_{n}\right\}, q_{n}(x) \in$ $\left\{1, \ldots, 2^{n}\right\}$. For $t \in H_{n}$, let

$$
C_{t}=\left\{z=\left(z_{i}\right) \in H_{n}^{n l_{n}} ; \exists i \leqslant n l_{n} ; z_{i}=t\right\} .
$$

Let $Z \subset H_{n}$. A point $x$ in $H_{n}^{n l_{n}}$ which has all its coordinates distinct and in $Z$ satisfies $\sum_{t \in Z^{1} C_{t}}(x) \geqslant n l_{n}$, so if card $Z \geqslant 2 n l_{n}$, we get

$$
Q_{n}\left(\left\{\sum_{t \in Z} 1_{C_{t}} \geqslant n l_{n}\right\}\right) \geqslant\left(\operatorname{card} Z / 2 \operatorname{card} H_{n}\right)^{n l_{n}} .
$$

Also, we note the following:

(51) Any family of $n l_{n}+1$ sets $C_{t}$ has empty intersection. For $x \notin F_{n}$, let

$$
W_{n}(x)=\left\{y \in L ; \forall q \leqslant n, y_{q}=x_{q}\right\} .
$$

For $x \in F_{n}$, let $W_{n}(x)=\left\{y \in L ; \forall q<n, y_{q}=x_{q}\right.$, and either $y_{n} \in H$ and $p_{n}(y)=$ $p_{n}(x)$ or $y_{n} \in M_{n}$ and $\left.y_{n} \in C_{x_{n}}\right\}$. For $x \in L$, define $\mathscr{B}(x)$ as the family of subsequences of $\left(W_{n}(x)\right)$.

We first show that condition $C_{\Phi}$ fails. Let $F=\bigcap F_{n}$, so $\mu(F) \geqslant \frac{1}{2}$. Fix $m \geqslant 1$. Define a Vitali cover $\mathscr{V}$ of $F$ by associating to $x \in F$ the sequence $\left(W_{n}(x)\right)_{n \geqslant 2 m+1}$. Let $\mathscr{F}$ be a finite family of $\mathscr{V}$-sets with $\mu\left(F \cap d_{\mathscr{F}}\right) \geqslant \frac{1}{4}$. Fix each $n$, let

$$
D_{n}=\bigcup\left\{A \in \mathscr{F} ; A \text { is of the type } W_{n}(x)\right\} \text {. }
$$

Since $d_{\mathscr{F}}=\bigcup_{n \geqslant m} D_{n}$, there is $n \geqslant 2 m+1$ with $\mu\left(F \cap D_{n}\right) \geqslant 2^{-n+1}$. Denote by $\Sigma_{n}$ the algebra of sets that depend only on the coordinates of rank $<n$. Let $G$ be the collection of the atoms $Y$ of $\Sigma_{n}$ such that

$$
\mu\left(F \cap D_{n} \cap Y\right) \geqslant 2^{-n} \mu(Y) .
$$

Then the union of $G$ has measure $\geqslant 2^{-n}$. Fix $Y$ in $G$ so that $\mu\left(F_{n} \cap D_{n} \cap Y\right) \geqslant$ $2^{-n} \mu(Y)$. For $x$ in $F \cap Y$, we have easily $\mu\left(W_{n}(x) \cap F_{n} \cap Y\right) \leqslant \mu(Y) / k_{n}$. It follows that there are at least $q=k_{n} 2^{-n}$ points $x^{1}, \ldots, x^{q}$ of $F \cap Y$ such that the sets $W_{n}\left(x^{1}\right), \ldots, W_{n}\left(x^{q}\right)$ are distinct and belong to $G$. Note that $q \geqslant 2 n l_{n}$, so (50) shows that

$$
\mu\left(\left\{\sum_{i \leqslant q} 1_{W_{n}\left(x^{j}\right)} \geqslant n l_{n}\right\} \cap Y\right) \geqslant b_{n}\left(2^{-2 n-1}\right)^{n l_{n}} \mu(Y)
$$

so

$$
\int_{Y} \Phi\left(e_{\mathscr{F}} / m\right) d P \geqslant \Phi\left(\left(n l_{n}-1\right) / m\right) b_{n}\left(2^{-2 n-1}\right)^{n l_{n}} \mu(Y) .
$$

By summation over $Y \in G$, from (49) and since $\left(n l_{n}-1\right) / m \geqslant 2 l_{n}$, we get $\left\|e_{\mathscr{F}}\right\|_{\Phi} \geqslant$ 1 .

We prove now that condition $F V_{\Phi}$ holds. Let $X \subset L, \mathscr{V}$ be a Vitali cover of $X$, and $\varepsilon>0$. To each $x \in X$ is associated a sequence $\left(W_{n_{q}}(x)\right)$ in $\mathscr{B}(x)$. For each $n$, let

$$
T_{n}=\left\{x \in X ; n \text { is of the type } n_{q}(x) \text { for some } q\right\} .
$$


Then for $x \in T_{n}, W_{n}(x)$ is a $\mathscr{V}$ set. Also, since $\mathscr{V}$ is a Vitali cover of $X$, we have $X \subset \limsup T_{n}$. For an atom $Y$ of $\Sigma_{n}$ and $j \leqslant k_{n}$, let us write

$$
Y_{j}=\left\{x \in Y \cap F_{n} ; p_{n}(x)=j\right\} .
$$

For each $n$, let $G_{n}$ be the family of the sets $Y_{j}$ for $Y$ an atom of $\Sigma_{n}, j \leqslant k_{n}$, such that

$$
\operatorname{card}\left\{r \leqslant 2^{n}, \exists x \in Y_{j} \cap T_{n}, q_{n}(x)=r\right\} \geqslant n .
$$

If $Y_{n} \notin G_{n}$, we have $\mu^{*}\left(Y_{j} \cap T_{n}\right) \leqslant n 2^{-n} \mu\left(Y_{j}\right)$. So, if $D_{n}$ is the union of $G_{n}$, we have

$$
\mu^{*}\left(T_{n} \backslash D_{n}\right) \leqslant \mu\left(L \backslash F_{n}\right)+n 2^{-n} \leqslant(n+1) 2^{-n} \text {. }
$$

This implies that $X \subset \limsup D_{n}$. Let us fix $\varepsilon>0$ and let $m$ large enough that $2^{-m+1}<\varepsilon$. For $n \geqslant m$, let

$$
S_{n}=\bigcup_{m-1 \leqslant r<n} F_{r} \backslash \bigcup_{m \leqslant r<n} D_{r} .
$$

Let $D_{n}^{\prime}=D_{n} \cap S_{n}$. Since $S_{n} \in \Sigma_{n}, D_{n}^{\prime}$ is the union of the sets $Y_{j}$ that it contains. Moreover, $P^{*}\left(X \cap \bigcap_{r \geqslant m-1} F_{r} \backslash \bigcup_{n \geqslant m} D_{n}^{\prime}\right)=0$, so $P^{*}\left(X \backslash \bigcup_{n \geqslant m} D_{n}^{\prime}\right)<\varepsilon$.

For $Y_{j}$ contained in $D_{n}^{\prime}$, let us pick, $n$ points $x^{1}, \ldots, x^{n} \in Y_{j} \cap T_{n}$ such that the numbers $q_{n}\left(x^{i}\right)$ are all different for $i \leqslant n$. Let $\xi_{Y, j}=(1 / n) \sum_{i \leqslant n} 1_{W_{n}\left(x^{i}\right)}$. This function is one on $Y_{j}$. Let $\xi_{n}$ denote the sum of the $\xi_{Y, j}$ for $Y_{j} \subset D_{n}^{\prime}$. Then $\xi_{n}=1$ on $D_{n}^{\prime}$. The point is that (51) implies that $\xi_{n} \leqslant l_{n}$, so $\int \Phi\left(\xi_{n}-\xi_{n} \wedge 1\right) d P \leqslant b_{n} \Phi\left(l_{n}\right) \leqslant 2^{-n}$. On the other hand, $\xi_{n}=1$ on $D_{n}^{\prime}$. Given $\varepsilon>0$, one can find $m^{\prime}$ with

$$
P^{*}\left(X \backslash \bigcup_{m \leqslant n \leqslant m^{\prime}} D_{n}^{\prime}\right) \leqslant \varepsilon .
$$

Since $\xi_{n}$ is zero outside $D_{n}^{\prime} \cup\left(F_{n-1} \backslash F_{n}\right)$, the functions $\xi_{n}$ have disjoint support, so if $\xi=\sum_{m \leqslant n \leqslant m^{\prime}} \xi_{n}$, we have $\xi=1$ on $\bigcup_{m \leqslant n \leqslant m^{\prime}} D_{n}^{\prime}$ while $\int \Phi(\xi-\xi \wedge 1) d P \leqslant 2^{-m+1} \leqslant$ $\varepsilon$. The proof is complete.

\section{REFERENCES}

1. K. Astbury, Order convergence of martingales, Trans. Amer. Math. Soc. 285 (1981), 495-510.

2. M. de Guzman, Differentiation of integrals in $\mathbf{R}^{n}$, Lecture Notes in Math., vol. 481, Springer-Verlag, 1975.

3. C. A. Hayes and C. Y. Pauc, Derivation and martingales, Springer-Verlag, 1970.

4. C. A. Hayes, Necessary and sufficient conditions for the derivation of integrals of $L_{\Psi}$-functions, Trans. Amer. Math. Soc. 223 (1976), 385-395.

5. K. Krickeberg, Convergence of martingales with a directed index set, Trans. Amer. Math. Soc. 83 (1956), 313-337.

6. J. Lindenstrauss and L. Tzafriri, Classical Banach spaces. II, Springer-Verlag, 1979.

7. A. Millet, Sur la caractérisation des conditions de Vitali par la convergence essentielle des martingales, C. R. Acad. Sci. Paris 287 (1978), 887-890.

8. A. Millet and L. Sucheston, On covering conditions and convergence, Measure Theory, Oberwolfach 1979, Lecture Notes in Math., vol. 794, Springer-Verlag, 1970, pp. 431-454.

9. __ A characterization of Vitali conditions in terms of maximal inequalities, Ann. Probab. 8 (1980), 339-349.

10. , On convergence of $L_{1}$-bounded martingales indexed by directed sets, Probab. Math. Statist. 1 (1980), 151-169.

11. K. Yosida and E. Hewitt, Finitely additive measures, Trans. Amer. Math. Soc. 72 (1952), 46-66.

12. A. C. Zaanen, Linear analysis, North-Holland, Amsterdam, 1953.

Equipe d'Analyse-Tour 46, Universite Paris VI, 75230 Paris Cedex 05, France

Department of Mathematics, Ohio State University, Columbus, Ohio 43210 